\title{
Emergence of microbial diversity due to cross-feeding interactions in a spatial model of gut microbial metabolism
}

\author{
Milan J. A. van Hoek and Roeland M. H. Merks' ${ }^{1} 2^{*}$ (I)
}

\begin{abstract}
Background: The human gut contains approximately $10^{14}$ bacteria, belonging to hundreds of different species. Together, these microbial species form a complex food web that can break down nutrient sources that our own digestive enzymes cannot handle, including complex polysaccharides, producing short chain fatty acids and additional metabolites, e.g., vitamin K. Microbial diversity is important for colonic health: Changes in the composition of the microbiota have been associated with inflammatory bowel disease, diabetes, obesity and Crohn's disease, and make the microbiota more vulnerable to infestation by harmful species, e.g., Clostridium difficile. To get a grip on the controlling factors of microbial diversity in the gut, we here propose a multi-scale, spatiotemporal dynamic flux-balance analysis model to study the emergence of metabolic diversity in a spatial gut-like, tubular environment. The model features genome-scale metabolic models (GEM) of microbial populations, resource sharing via extracellular metabolites, and spatial population dynamics and evolution.
\end{abstract}

Results: In this model, cross-feeding interactions emerge readily, despite the species' ability to metabolize sugars autonomously. Interestingly, the community requires cross-feeding for producing a realistic set of short-chain fatty acids from an input of glucose, If we let the composition of the microbial subpopulations change during invasion of adjacent space, a complex and stratified microbiota evolves, with subspecies specializing on cross-feeding interactions via a mechanism of compensated trait loss. The microbial diversity and stratification collapse if the flux through the gut is enhanced to mimic diarrhea.

Conclusions: In conclusion, this in silico model is a helpful tool in systems biology to predict and explain the controlling factors of microbial diversity in the gut. It can be extended to include, e.g., complex nutrient sources, and host-microbiota interactions via the intestinal wall.

Keywords: Flux-balance analysis with molecular crowding, Dynamic multi-species metabolic modeling, Intestinal microbiota, Multiscale modeling, Compensated trait loss, Microbial communities

\section{Background}

The human colon is a dense and diverse microbial habitat, that contains hundreds of microbial species [1]. These species together form a community that breaks down complex polysaccharides into monosaccharides, which are then fermented further into short chain fatty acids (SCFAs) that are taken up by the host [2]. The

\footnotetext{
*Correspondence: merks@cwi.nl

'Life Sciences Group, Centrum Wiskunde \& Informatica, Science Park 123, 1098 XG Amsterdam, The Netherlands

${ }^{2}$ Mathematical Institute, Leiden University, Niels Bohrweg 1, 2333 CA, Leiden, The Netherlands
}

composition of the intestinal microbiota and the topology of the community-level metabolic network formed by it [3] are associated with health and disease. For example, the microbiota produces the short-chain fatty acid butyrate, which has been proposed to lower the risk for colon cancer [2]. Inflammatory bowel disease (IBD) and obesity are correlated with gain or loss of enzymes in the periphery of the network [3], suggesting that in obese persons and in IBD patients the microbiota produces a different set of metabolic end-products. Topological analysis further found indications that microbiota of obese individuals have a more diverse set of enzymes to 
extract energy from the diet [3]. Patients with diarrheapredominant irritable bowel syndrome show large temporal shifts in the composition of the microbiota [4].

The most important source of bacterial diversity in the colon is probably due to metabolic interactions between bacteria [5]. The main nutrient sources entering the colon are non-degraded polysaccharides, including resistant starch and cellulose, oligosaccharides, proteins and simple sugars [6]. In addition to these exogenous sources of sugar, the colonic epithelium secretes mucins, which are an important nutrient source for the microbiota [6].

In this paper we ask what mechanisms are responsible for the diversity of the gut microbiota. The structured environment and the diversity of undigested nutrient sources (e.g., complex polysaccharides, e.g., found in food fibers) found in the gut have been shown to sustain diverse microbial communities [2, 7]. Interestingly, however, diverse ecosystems can also arise in homogeneous environments with only one primary resource [8-12]. For example, glucose-limited, continuous cultures of E. coli reproducibly evolve acetate cross-feeding within about 100 generations (see Ref. [11] and references therein). In these experiments, one subpopulation enhances its glucose uptake efficiency and secretes acetate as a waste product. The acetate then provides a niche for a second strain that can grow on low concentrations of acetate.

Mathematical modeling can help understand under what conditions such cross-feeding and diversification can emerge in homogeneous environments. In their isologous diversification model, Kaneko and Yomo [13, 14] studied sets of identical, chaotically oscillating metabolic networks that exchange metabolites via a common, shared medium. Although small populations of oscillators will easily synchronize with one another, larger populations will break up in specialized, synchronized sub-populations. Mathematical modeling has also given insight into the conditions that make specialization and cross-feeding beneficial from an evolutionary point of view. For example, cross-feeding can evolve if there exists a trade-off between uptake efficiency of the primary and secondary nutrient source [15], or if a trade-off exists between growth rate and yield [16]. In absence of such metabolic trade-offs, cross-feeding can evolve if the enzymatic machinery required to metabolize all available nutrients is so complex that distributing enzymes across a number of species or strains becomes the more probable, 'easier' evolutionary solution [17].

These initial mathematical models included simplified or conceptual models of metabolism. More recently, it has become feasible to construct models of microbial communities based on genome-scale metabolic network models (reviewed in Ref. [18]). In these models, multiple species of bacteria interact with one another by modifying a common pool of metabolites. One class of models optimizes the bacterial and community growth rates in parallel, assuming flux-balance of whole community at once [19] or iteratively within the individual bacteria and at community level [20]. Such approaches can also include dynamic changes of the community-level constraints, including extracellular concentrations of metabolites [21].

To also capture the emergent population dynamics of bacterial communities due to secretion and uptake of metabolites by the bacteria, (static optimizationbased) dynamic flux-balance analysis (dFBA) has been introduced [22]. These couple the optimization-based flux-balance analysis (FBA) approach for modeling intracellular metabolism, with an ordinary-differential equation model (ODE) for modeling the metabolite concentrations in the substrate. These community models more closely approximate microbial metabolism than the initial, more abstract models, such that the results can be compared directly to experimental observations. For example, Tzamali and coworkers [23] used multispecies dFBA to compare the performance of metabolic mutants of E. coli in batch monoculture versus its performance in co-culture with an alternative mutant. Their model predicted co-cultures that were more efficient than their constituent species. Louca and Doebeli [24] proposed methodology to calibrate the bacterial models in such dynamic multispecies FBA approaches to data from experimental monocultures. By coupling these calibrated dynamical models of isolated strains of E. coli, the framework could reproduce experimentally observed succession of an ancestral monoculture of $E$. coli by a crossfeeding pair of specialists. Because these models assume direct metabolic coupling of all species in the model via the culture medium, the model best applies to well-mixed batch culture systems or chemostats. The more recent coupled dynamic multi-species dFBA and mass transfer models [18, 25-27], or briefly, spatial dFBA (sdFBA) models are more suitable for modeling the gut microbiota. These spatial extensions of the multispecies dFBA approach couple multiple dFBA models to one another via spatial mass transport models (based on numerical solutions of partial-differential equations), such that bacteria can exchange metabolites with their direct neighbors.

In order to explore whether and under which circumstances a diverse microbial community can arise from a single nutrient source in the gut, here we extended the sdFBA approach to develop a multiscale model of collective, colonic carbohydrate metabolism and bacterial population dynamics and evolution in a gut-like geometry. To this end, we combined spatial models of population dynamics with genome-scale metabolic models (GEMs) for individual bacterial species and a spatial mass transport model. In addition to the sdFBA approaches, we extended the model with an "evolutionary" component, in order to allow for unsupervised diversification of the 
microbial communities. We inoculate the metabolic system with a meta-population of bacteria containing a set of available metabolic pathways. When, depending on the local availability of nutrients, the bacterial population expands into its local neighborhood the metapopulation gains or looses metabolic pathways at random. We find that spatially structured, microbial diversity emerges spontaneously in our model starting from a single resource. This diversity depends on interspecies crossfeeding interactions.

\section{Results}

A full multiscale model of the metabolism of the human gut would need to include around $10^{14}$ individual bacteria belonging to hundreds of bacterial species, for which in many cases curated GEMs are unavailable. We thus necessarily resorted to a more coarse-grained approach, while maintaining some level of biological realism by constructing the model based on a validated, genome-scale metabolic network model of Lactobacillus plantarum [28]. Figure 1 gives an overview of the workflow of the paper. We first (1) constructed a metabolic model representing a subset of the gut microbiota, which we used for the AFBA model (2). We then asked to what extent cross-feeding can emerge in large communities of interacting and diversified bacteria, such as those found in the colon, using a dynamic multi-species metabolic modeling (DMMM) approach [18, 23, 29], which is an extension of the dynamic flux-balance analysis (dFBA) method
$[22,30]$. To this end, we constructed a well-mixed model of a bacterial consortium (3), by coupling 1000 of the dFBA models via a common, external exchange medium that allowed the bacteria to exchange a subset of the metabolites in the GEM. We initiated the exchange medium with a pulse of glucose, then observed the turnover of glucose into a series of short-chain fatty acids (4), and quantified cross-feeding (5): the extent to which the bacteria exchanged metabolites via the common medium. Next we asked to what extent spatially diversified microbial communities can emerge in a tube-like environment (6), if the microbial communities are allowed to specialize to the local availability of metabolites. In the spatial model, the GEMs inside the bacteria were allowed to evolve. After running the model for a fixed time, we quantified how much the GEMs had diversified and performed local cross-feeding (7) and to what extent they had locally changed the external concentrations of metabolites (8), leading to stratification and niche formation.

\section{Construction of a metabolic model representing a subset of the gut microbiota}

We first constructed a hypothetical, but biologicallyrealistic "supra-organism" model $[3,31]$, called "metabacterium" here, that represents a sample of the gut microbial community in a single metabolic network model. For this preliminary, explorative study we used a GEM of Lactobacillus plantarum [28], a resident of the colon and a strain widely used for probiotics, and extended

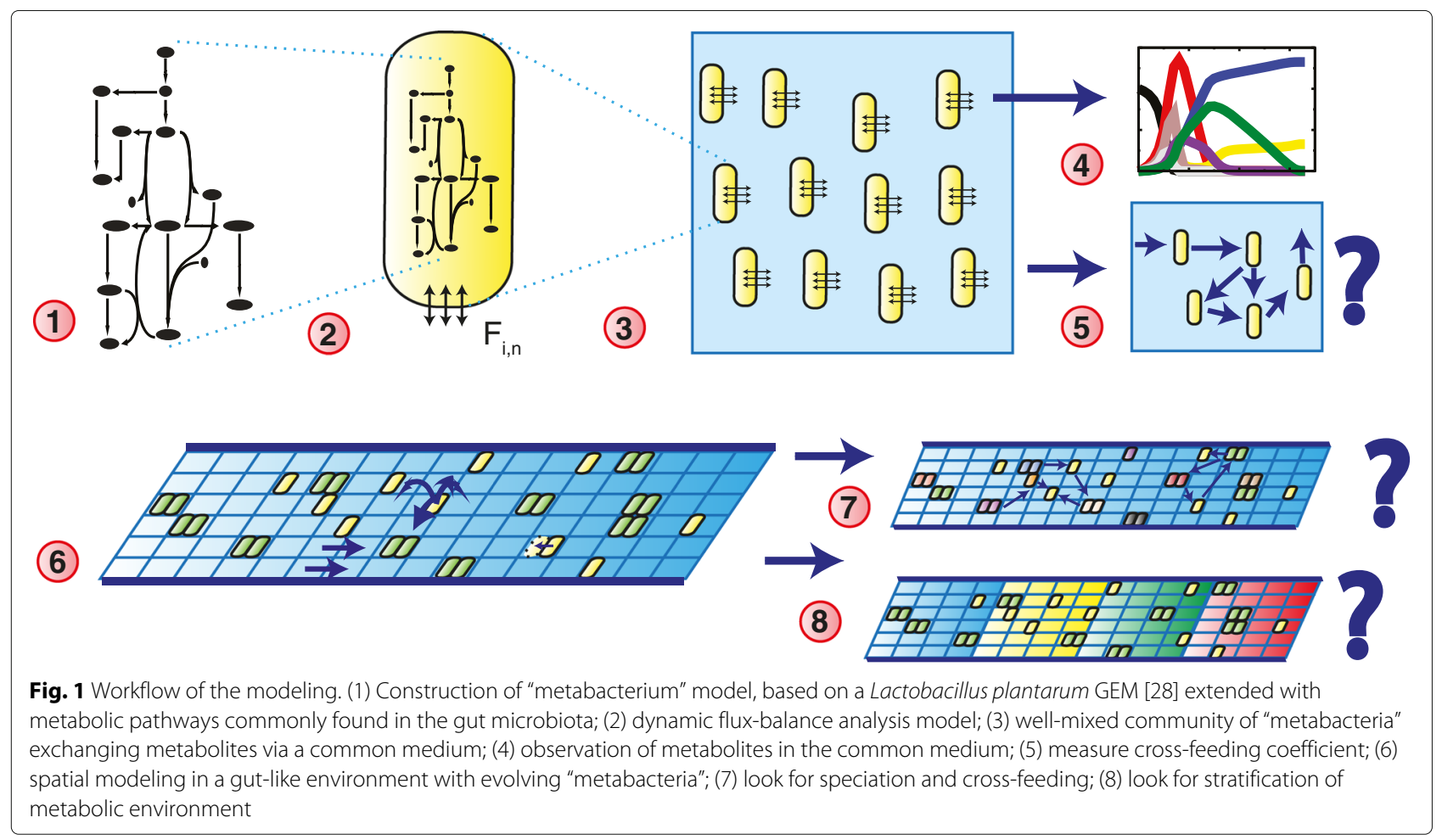


it with four key metabolic pathways of the intestinal microbial community: (1) propionate fermentation, (2) butyrate fermentation, (3) the acrylate pathway and (4) the Wood-Ljungdahl pathway. In future versions of our framework this network could be replaced by metabolic network models derived from metagenomic data [3] as they become available. The current, simplified network contains 674 reactions (Supplementary File 1), and compares well with consensus metabolic networks of carbohydrate fermentation in the colon [32,33]. For a schematic overview of the key pathways including in the metabolic network, see Fig. 2a.

The uptake and excretion rates of genome-scale metabolic networks can be calculated using constraintbased modeling. To represent diauxic growth, i.e., byproduct secretion as a function of extracellular metabolite concentrations, we used an extension of FBA called Flux Balance Analysis with Molecular Crowding (FBAwMC) [34]. FBAwMC correctly predicts diauxic growth and the associated secretion of by-products in micro-organisms including E. coli, Saccharomyces cerevisiae [35], and L.plantarum [36]. As an additional, physiologicallyplausible constraint FBAwMC assumes that only a finite number of metabolic enzymes fits into a cell, with each enzyme having a maximum metabolic turnover, $V_{\max }$. For each reaction, FBAwMC requires a crowding coefficient, defined as the enzymatic volume needed to reach unit flux through that reaction. Each reaction is assigned a "crowding coefficient", a measure of the protein cost of a reaction: Enzymes with low crowding coefficients have small molecular volume or catalyse fast reactions. Given a set of maximum input fluxes, FBAwMC predicts the optimal uptake and excretion fluxes as a function of the extracellular metabolite concentrations.

As FBAwMC optimizes growth rate, not growth yield as in standard FBA, it predicts a switch to glycolytic metabolism at high glucose concentrations at which faster metabolism is obtained with suboptimal yield. Its accurate prediction of diauxic growth together with by-product secretion as a function of extracellular metabolite concentrations make FBAwMC a suitable method for a microbial community model.

\section{Metabolic diversity causes cross-feeding in a well-mixed system}

To study the extent of cross-feeding emerging already from a non-evolving metabolic community of "metabacteria", we first set up a simulation of 1000 interacting metapopulations, where each subpopulation was initiated with a set of crowding coefficients selected at random from an experimentally determined distribution of crowding coefficients of Escherichia coli [35, 36], for lack of similar data sets for L. plantarum. The simulation was initiated with pure glucose and was ran under anaerobic conditions. We then performed FBAwMC on all 1000 metapopulations, optimizing for ATP production rate as a proxy for growth rate. This yielded 1000 sets of metabolic input and output fluxes, $F_{i}$, and growth rates, $\mu_{i}$ for all 1000 metapopulations. These were used to update the extracellular concentrations, $\vec{M}$ and metapopulation sizes, $X_{i}$, by performing a single finite-difference step of $[23,29]$

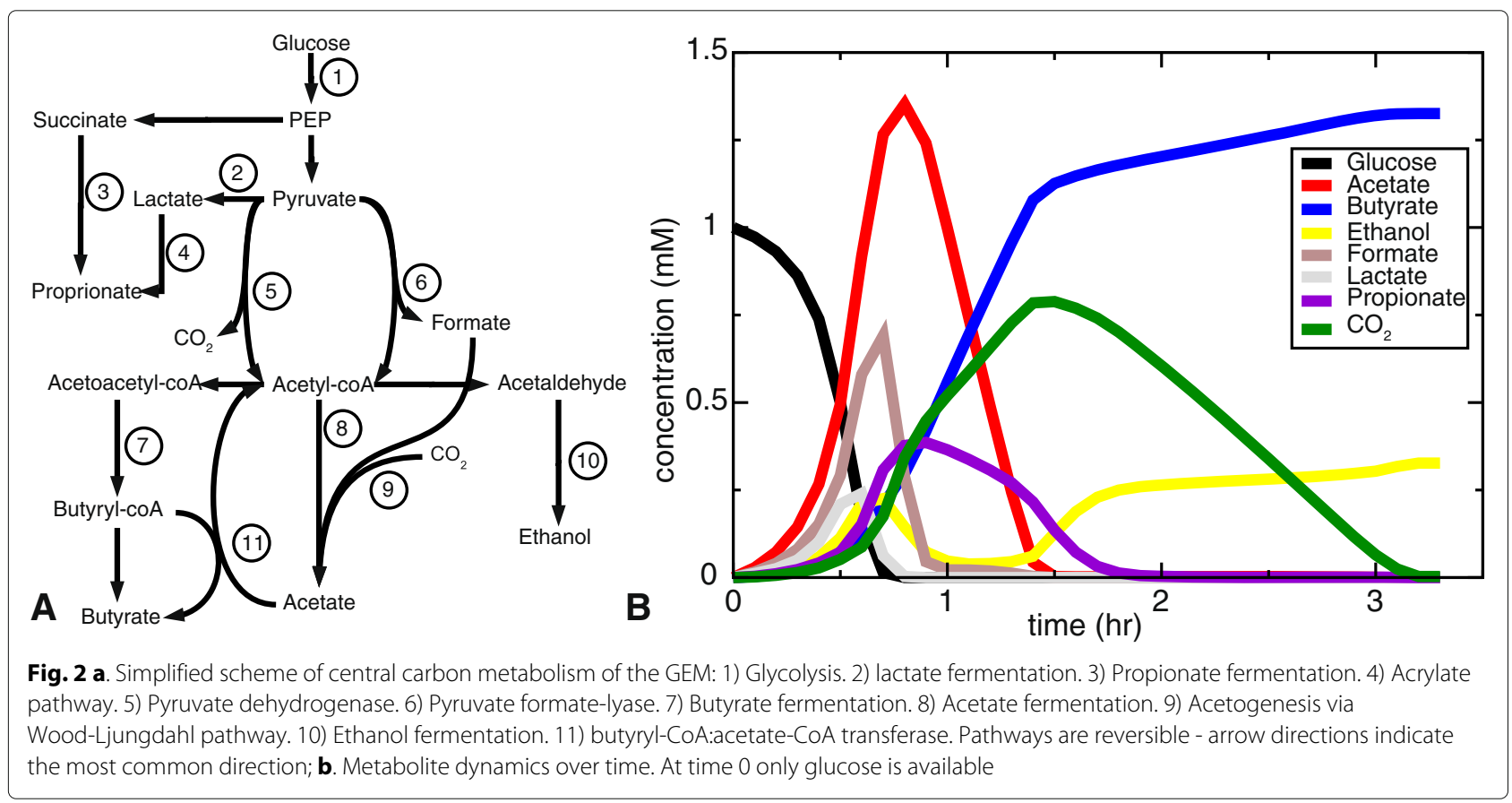




$$
\frac{d \vec{M}}{d t}=\sum_{i} X_{i} \vec{F}_{i}
$$

and

$$
\frac{d X_{i}}{d t}=\mu_{i} X_{i}
$$

with a timestep $\Delta t=0.1 \mathrm{~h}$. After updating the environment in this way, we performed a next time simulation step.

Figure $2 \mathrm{~b}$ shows how, in the simulation, the metabacteria modified the environment over time. The secondary metabolites that were produced mostly are acetate, butyrate, carbon dioxide, ethanol, formate, lactate and propionate. This compares well with the metabolites that are actually found in the colon [37] or in an in vitro model of the colon [38]. In the first $30 \mathrm{~min}$ of the simulation, the initial pulse of glucose is consumed, and turned over into acetate (red), lactate (grey), formate (brown), and ethanol (yellow). These are then consumed again, and turned over into proprionate (purple) via pathways 3 and 4 (Fig. 2a) and into butyrate (blue) via pathways 7 and $11 . \mathrm{CO}_{2}$ is also increasing due to the turnover of pyruvate into acetyl co-A via pathway 5 (pyruvate dehydrogenase). After about two hours of simulated time, proprionate and $\mathrm{CO}_{2}$ levels drop again due to the production of butyrate (blue): proprionate is consumed reversing reaction 3 and $4 ; \mathrm{CO}_{2}$ is consumed in pathway 9 that produces acetate from formate. The conversion of acetate back to acetyl-coA then drives the production of butyrate; a surplus of acetyl-coA is turned over into acetaldehyde and ethanol in pathway 10. Interestingly, formate and $\mathrm{CO}_{2}$ are produced at the same time; this rarely occurs in any single organism but does occur in this microbial consortium.

To test to what extent these results depend on the ability of the individual FBAwMC models to represent metabolic switching and overflow metabolism $[34,36]$, we also simulated the model using standard flux-balance analysis [39]. In this case, all glucose was converted into ethanol, whereas lactate and propionate did not appear in the simulation (Additional file 1: Figure S1). To test to what extent the results rely on cross-feeding, we also checked if any of the single-species simulations could also produce so many metabolites. Out of 100 single-species simulations none produced as many or more excreted metabolite species than the interacting set of species.

\section{Quantification of cross-feeding}

Most of the metabolites were only transiently present in the medium, $\vec{M}$, suggesting that the metabolites were re-absorbed and processed further by the bacteria. To quantify the amount of such cross-feeding in the simulations, we defined a cross-feeding factor, $C(i)$, with $i$ a species identifier. Let

$$
\begin{aligned}
& F_{\text {up tot }}(i, j) \equiv \int_{t=0}^{t_{\max }} B(n, t) F_{\text {up }}(i, j, t) d t \\
& F_{\text {ex tot }}(i, j) \equiv \int_{t=0}^{t_{\max }} B(n, t) F_{\text {ex }}(i, j, t) d t
\end{aligned}
$$

be the total amount of metabolite $j$ that species $i$ consumes and excretes during the simulation. $B(i, t)$ here equals the biomass of species $i$ at time $t$. The amount of carbon species $i$ gets via cross-feeding then equals,

$$
\begin{aligned}
C(i)= & \sum_{j} c_{C}(j) \max \left(F_{\text {up,tot }}(i, j)-F_{\text {ex,tot }}(i, j), 0\right) \\
& -6 F_{\text {up,tot }}(i, \text { glucose }) .
\end{aligned}
$$

Here, $c_{\mathrm{C}}(j)$ is the molar amount of carbon atoms per mol metabolite $j$ (e.g., $c_{\mathrm{C}}$ (glucose) $=6$ ). If species $i$ during the fermentation consumes more of metabolite $j$ than it has produced, species $i$ has cross-fed on metabolite $j$. We subtract the amount of glucose from the sum, because glucose is the primary nutrient source that is present at the start of the simulation. Now we can calculate the total amount of carbon the population acquires via cross-feeding, relative to the total amount of carbon taken up by the population

$$
C_{\text {rel }}=\frac{\sum_{i} C(i)}{\sum_{i} \sum_{j} c_{C}(j) F_{\text {up }, \text { tot }}(i, j)} .
$$

If $C_{\text {rel }}=0$, there is no cross-feeding. In that case, every species only consumes glucose as carbon source or only consumes as much carbon from other metabolites as it has secreted itself. Conversely, if $C_{\text {rel }}=1$ all carbon the species has consumed during the simulation is from non-glucose carbon sources the species has excreted itself. For the whole simulation $C_{\text {rel }}=0.39 \pm 0.02$, indicating that $39 \%$ of all carbon consumed by the bacteria comes from cross-feeding. Cross-feeding was largest on lactate, $\mathrm{CO}_{2}$, acetate, ethanol, formate and propionate. Many of these metabolites are known to be involved in bacterial cross-feeding in the colon or cecum (for interconversion between acetate and lactate, see Ref. [40]; and for interconversion between acetate and butyrate in the murine cecum, see Ref. [41]). In the original L. plantarum model we also find cross-feeding, but only on lactate and acetaldehyde (Additional file 2: Figure S2). Taken together, in agreement with previous computational studies that showed cross-feeding in pairs of interacting E. coli [23], these simulations show that cross-feeding interactions occur in coupled dynamic FBAwMC models.

\section{Spatially explicit, evolutionary model}

The well-mixed simulations showed that cross-feeding appears in populations of interacting metabacterial metabolic networks. However, this does not necessarily imply microbial diversity, because it is possible that the same metabacterium secretes and reabsorbs the same metabolites into the substrate, in which case there would 
be no true cross-feeding. Furthermore, the previous section did not make clear whether cross-feeding will be ecologically stable under conditions where subpopulations of the supra-organisms are lost. In a spatially explicit model, cross-feeding possibly arises more easily and is more easy to detect, as different metabolic functions can be performed at different locations [42]. We therefore developed a spatially explicit, multiscale evolutionary model of gut microbial metabolism. We initiate the simulation with a population of metapopulations of bacteria that can perform all metabolic functions under anaerobic conditions, just as in the well-mixed simulation. We then let the systems evolve and study if meta-populations of bacteria with specific metabolic roles evolve.

\section{Model description}

Figure 3 sketches the overall structure of our model. The model approximates the colon as the cross-section of a $150 \mathrm{~cm}$ long tube with a diameter of $10 \mathrm{~cm}$. The tube is subdivided into patches of $1 \mathrm{~cm}^{2}$, each containing a uniform concentration of metabolites, and potentially a metapopulation of gut bacteria (hereafter called "metabacterium") (Fig. 3a). Each metabacterium represents a small subpopulation (or 'metapopulation') of gut bacteria with diverse metabolic functions, and is modeled using a metabolic network model containing the main metabolic reactions found in the gut microbiota, as described above (Fig. 2a). Based on the local metabolite concentrations, $\vec{c}(\vec{x}, t)$, the metabolic model delivers a set of exchange fluxes $F_{\mathrm{i}, \mathrm{n}}$ and a growth rate, $\mu(\vec{x})$, which is assumed to depend on the ATP production rate (Fig. 3b; see "Methods" for detail). The metabolites disperse to adjacent patches due to local mixing, which we approximate by a diffusion process (Fig. 3c), yielding

$$
\frac{d \vec{c}(\vec{x}, t)}{d t}=\vec{F}(\vec{x}, t) B(\vec{x}, t)+\frac{D}{L^{2}} \sum_{\vec{i} \in \mathrm{NB}(\vec{x})}(\vec{c}(\vec{i}, t)-\vec{c}(\vec{x}, t)),
$$

where $\vec{F}(\vec{x}, t)$ is the flux of metabolites between the medium and the metabacterium, and the sum runs over

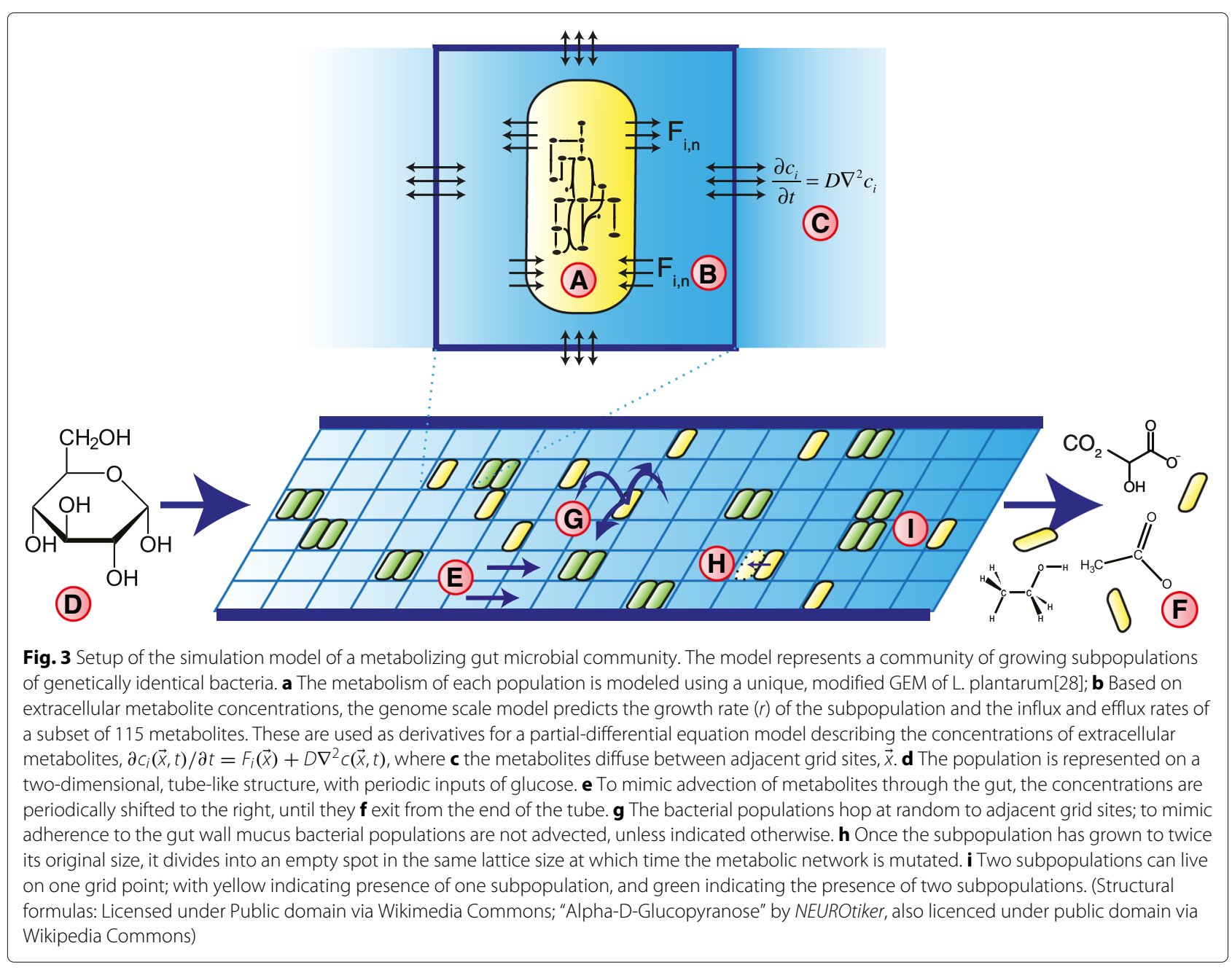


the four nearest neighbors $\mathrm{NB}(\vec{x})$; dispersion is approximated by Fick's law, where $D$ is a diffusion coefficient and $L=1 \mathrm{~cm}$ the interface length between two adjacent patches. The local density of metabacteria, $B(\vec{x})$ is given by

$$
\frac{d B(\vec{x}, t)}{d t}=\mu(\vec{x}, t) B(\vec{x}, t) .
$$

To mimic meals, a pulse of glucose of variable magnitude enters the tube once every eight hours (Fig. 3d). The metabolites move through the tube via a simplified model of advection: At regular intervals, all metabolites are shifted one patch (Fig. 3e). Metabolites continuously leave the tube at the end through an open boundary condition (Fig. 3f). To mimic peristaltic movements that locally mix the gut contents together, metabacteria randomly hop to adjacent lattice sites (Fig. 3g) and leave the gut only via random hops over the open boundary condition. In a subset of simulations, accelerated bowel movements are simulated by advecting the metabacteria together with the metabolites. To a first approximation, the boundaries are impermeable to the metabolites, a situation reflecting many in vitro models of the gut microbiota (reviewed in Ref. [43]); later versions of the model will consider more complex boundary conditions including absorption of metabolites [44].

When the local biomass in a patch, $B(\vec{x}, t)$, has grown to twice its original value, the metapopulation expands into the second position on the grid point (Fig. 3h). To mimic a local carrying capacity, the metapopulation does not spread out or grow any further if both positions in the patch are occupied. In the visualizations of the simulations, full patches are shown in green, singly occupied patches are shown in yellow, and empty patches are shown in black (Figs. 3i and 4). During expansion, changes in the relative abundance of species may enhance or reduce the rate of particular reactions, or even delete them from the metapopulation completely. Similarly, metabolic reactions can be reintroduced due to resettling of metabolic species, e.g., from the gut wall mucus [45]. To mimic such changes in species composition of the metapopulation, during each expansion step, we delete enzymes from the metabolic network at random, reactivate enzymes at random, or randomly change crowding coefficients such that the metapopulation can specialize on one particular reaction or become a generalist.

The crowding coefficients, as they appear in the fluxbalance analysis with molecular crowding (FBAwMC) method that we used for this model, give the minimum cellular volume filled with enzymes required to generate a unit metabolic flux; they are given by the $V_{\max }$ of the enzyme and enzyme volume [34]. Equivalently, in our metapopulation model, the crowding coefficient of a reaction is the minimum intracellular volume averaged over all bacteria in the patch that must be filled with enzymes in order to generate a unit flux through the reaction. It depends on the density of the enzyme in the bacteria and also on the corresponding values of $V_{\max }$. Because the $V_{\max }$ of a reaction can differ orders of magnitudes between species (see for example the enzyme database BRENDA [46]), the evolutionary dynamics in our model could drive the metabacteria to reduce all crowding coefficients concurrently, producing a highly efficient generalist. To introduce a biologically more realistic trade-off between metabolic rate and cost in terms of volume, we therefore included an experimentally observed trade-off between growth rate and growth yield among microorganisms [47, 48]: Micro-organisms that grow fast have low growth yield and vice versa. We take this trade-off into account explicitly by assuming a maximal growth rate given the carbon uptake rate of the cells. This trade-off prevents the metabacteria from growing infinitely fast by mutating their crowding coefficients.

As an initial condition, we distribute metabacteria over the grid, each containing all available metabolic reactions, i.e., each metabacterium initially contains all bacterial "species" that the complete metabacterium represents. To reflect variability in the relative abundances of the bacterial species in each metabacterium the crowding coefficients are drawn at random from an experimental distribution as described above (Fig. 3a).

\section{Evolution of diversity due to metabolic cross-feeding}

To evaluate the behavior of our model, we performed ten independent simulations. These show largely similar

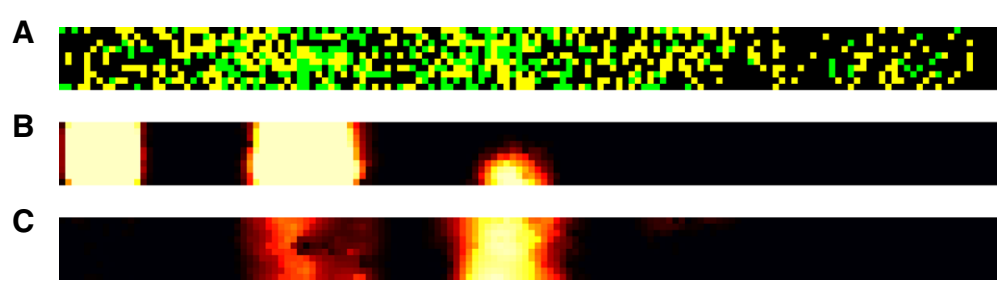

Fig. 4 Screenshot of the spatially explicit model. The proximal end of the colon is on the left, the distal end on the right. Thus, nutrients flow from left to right. a Cells on the grid. At maximum 2 cells can be on the same grid point. Yellow:one cell present, green: 2 cells present. (b) Glucose concentration. Black: low concentrations, white: high concentrations. (c) Formate concentrations. In total, 115 extracellular metabolites are taken into account in the model 
phenomenology; therefore we will first describe the progression of one representative simulation in detail, and then discuss differences with the other simulations. Figure 5a shows the average number of metabolic reactions present in the metabacteria over time in the simulation. At $t=0$ all metabacteria still have all 674 reactions, but over time the number of available reactions gradually drops to below 200 . This reduction of the number of metabolic genes could indicate a homogeneous population that is specialized, e.g., on fermentation of glucose where most of the metabolic network is not used. An alternative explanation is that each of the metapopulation retains a subset of the full network, an indication of crossfeeding. The amount of cross-feeding will likely change over the tube: The metabacteria in the front have direct access to glucose, whereas the metabacteria further down in the tube may rely on the waste-products of those in front. We therefore determined a temporal average of the cross-feeding factors, $C_{r e l}$ (Eq. 5), at each position in the tube over $t=3500$ to $t=4000$, a time range at which most genes have been lost. The first observation to note is that in the spatial evolutionary simulations, the average cross-feeding factor $C_{\text {rel }}$ has a higher value than in the well-mixed simulations. In this particular simulation, the spatial average cross-feeding factor at $t=4000$ is $C_{\text {rel }}=0.65 \pm 0.09$, compared with $C_{\text {rel }}=0.39 \pm 0.02$ in the well-mixed case $(n=10)$. The cross-feeding factor for individual cells $(C(i)$, Eq. 4), showed large population heterogeneity. As Fig. 5b shows, the cross-feeding factor in the tube front is close to 0 , indicating the presence of primary glucose consumers, while cross-feeding slowly increases towards the distal end until it almost reaches 1 , indicating complete cross-feeding. Thus in the proximal end the bacteria rely mostly on the primary nutrient source, while near the distal end cells of the tube rely on cross-feeding. This observation is consistent for all simulations (see Additional file 3: Figure S3).

\section{Emergence of metabolic stratification}

We next investigated the mechanism by which such crossfeeding emerges in the simulation. Additional file 4: Figure S4 plots the metabolite concentrations over evolutionary time for the simulation of Fig. 5. In this particular simulation, the concentrations of formate and lactate initially rise rapidly, after which they drop gradually. The butyrate concentrations increase over evolutionary time. In all simulations, the metabolite concentrations change gradually, but not necessarily following the same temporal pattern.

Figure 6 shows the spatial distribution of a set of key metabolites averaged over $2000 \mathrm{~h}$ to $4000 \mathrm{~h}$ of the representative simulation. Interestingly, the flow of metabolites through the colon in interaction with the bacterial population creates a spatially structured, metabolic environment. The proximal end is dominated by the primary carbon source glucose (Fig. 6a), with the peak in the average glucose concentration due to the periodic glucose input. Further down in the tube we find fermentation products, including lactate and ethanol, whereas the distal end contains high levels of acetate and $\mathrm{CO}_{2}$, showing that the metabacteria convert the glucose into secondary metabolites. Among these secondary metabolites, the levels of acetate (Fig. 6b), ethanol (Fig. 6e), formate (Fig. 6f), lactate (Fig. 6g) and propionate (Fig. 6h) drop towards the distal end off the tube, so they are further metabolized by the metabacteria. In this particular simulation, butyrate and $\mathrm{CO}_{2}$ are not consumed and their concentrations increase monotonically towards the end. The small drop at the very distal end is caused by the metabolite outflow. The profiles of the other simulations were consistent with this representative simulation (Additional file 5: Figure S5). In all simulations, the proximal end was dominated by glucose. Further towards the end of the tube, zones of fermentation products developed as in the representative simulation, but the precise location of each product was different and not all products were present. Most notably, in two out
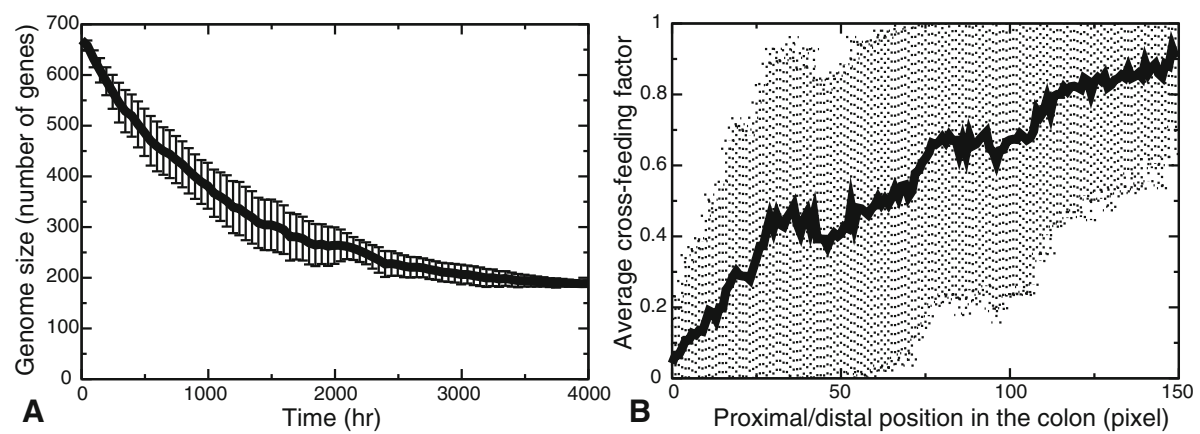

Fig. 5 Outcome of the evolutionary simulations. a population average and standard deviation of the number of enzymatic reactions ("genome size") over time. b Population average and standard deviation of the cross-feeding factor $\mathrm{Cn}$ as a function of the position in the colon. The averages and standard deviation are over the vertical dimension and are calculated over the final part of the simulation, from $3500 \mathrm{~h}$ until $4000 \mathrm{~h}$. For the graphs of the other simulations, see Additional file 3: Figure S3 

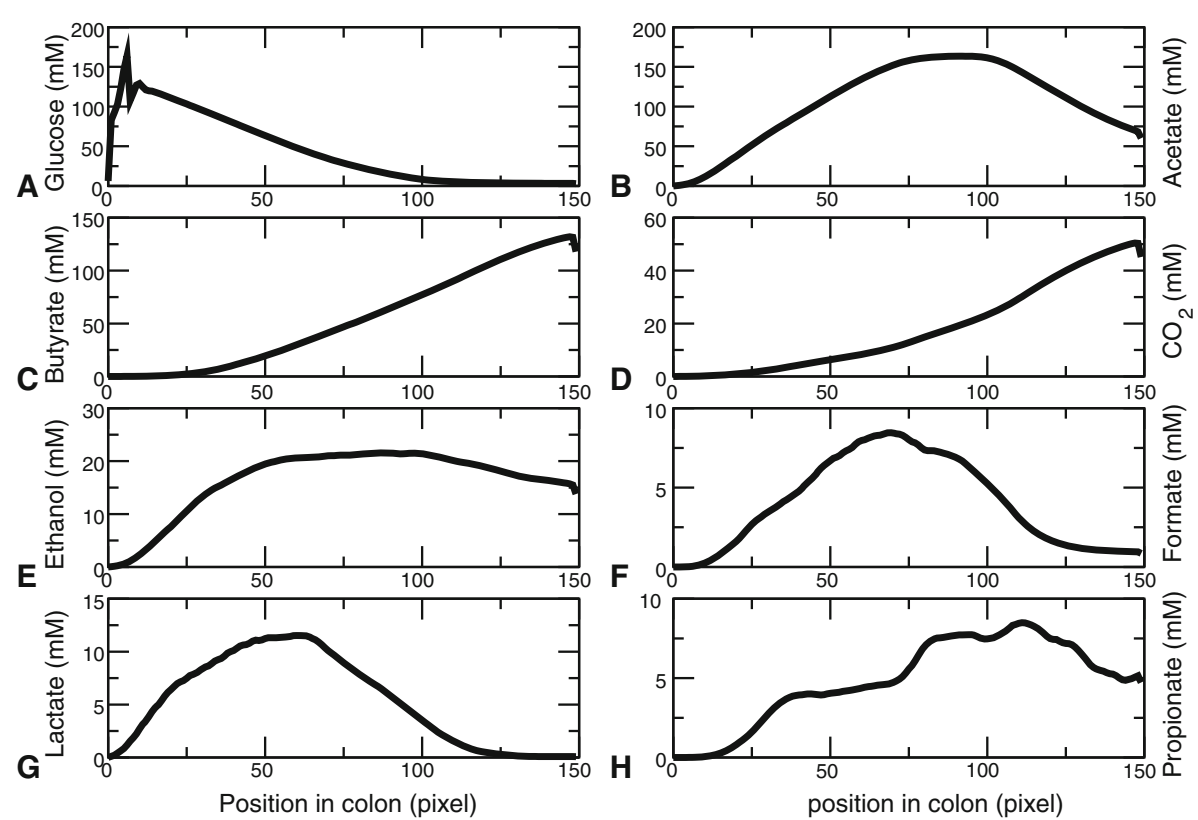

Fig. 6 Average metabolite concentrations along the colon. Average are taken over the second half of the simulation (2000hrs-4000hrs). a Glucose. b Acetate. c Butyrate. $\mathbf{d ~} \mathrm{CO}_{2}$. e Ethanol. fFormate. $\mathbf{g}$ Lactate. $\mathbf{h}$ Propionate

of ten simulations, butyrate was absent and in two other simulations proprionate was absent. Also, in three out of ten simulations lactate was more confined to the front of the tube (up to around 50 sites) than in the representative simulation.

\section{Metabacteria specialize on local metabolic niches}

These results demonstrate that the metabacteria spatially structure their metabolic environment, generating a stratified structure of metabolic "niches" along the tube, each offering a separate set of metabolites. Therefore, we next asked if this environmental structuring gives rise to metapopulations uniquely adopted to the microenvironment. We took computational samples of all metabacteria found in the tube between $3500 \mathrm{~h}$ and $4000 \mathrm{~h}$, to average out the variations at the short timescale. We tested the growth rate of these samples (consisting of on average $n \approx 1100$ metabacteria) in six, homogeneous metabolic environments, containing uniform concentrations of pure (1) glucose, (2) acetate, (3) formate, (4) lactate, and (5) propionate, and (6) a mixture of of $\mathrm{CO}_{2}$ and $\mathrm{H}_{2}$. Figure 7 shows the average and standard deviation of the growth rates of the metabacteria in each of these six environments, as a function of the position from which they were sampled from the tube. Strikingly, the metabacteria near the distal end of the tube have lost their ability to grow on glucose (Fig. 7a), indicating that they have specialized on secondary metabolites, including acetate (Fig. 7b) and lactate (Fig. 7e). Interestingly, in support of the conclusion that the metabacteria specialize on the metabolic niches generated by the population as a whole, the metabacteria sampled from the distal end on average grow faster on acetate and lactate than the metabacteria sampled from the front of the tube. Acetate and lactate are produced in the proximal colon and flow to the distal part of the tube where the metabacteria can metabolize it; in the front of the tube acetate and lactate concentrations are lower, such that neutral drift effects can safely remove the corresponding metabolic pathways from the metabacteria. Remarkably, the metabacteria also grow on $\mathrm{CO}_{2}$, because of the presence of hydrogen gas, that allows growth on $\mathrm{CO}_{2}$ via the Wood-Ljungdahl pathway [49]. To further characterize the alternative metabolic modes occurring in the model, we clustered the population present at the end of the simulation $t=4000 \mathrm{~h}$ with respect to their maximum growth rates in the six environments (Fig. 8). Clearly, different metabolic "species" can be distinguished. One "species" can metabolize glucose, a second "species" can metabolize most secondary metabolites and a third "species" has specialized on acetate. Thus in our simulation model a number of functional classes appear along the tube, each specializing on its own niche in the full metabolic network.

\section{Increased flux through the tube makes diversity collapse}

From the results in the previous section, we conclude that the inherent spatial structuring of the colon results in separate niches. This allows the population to diversify, such that different "species" have different metabolic tasks. A recent population-wide metagenomics study of 

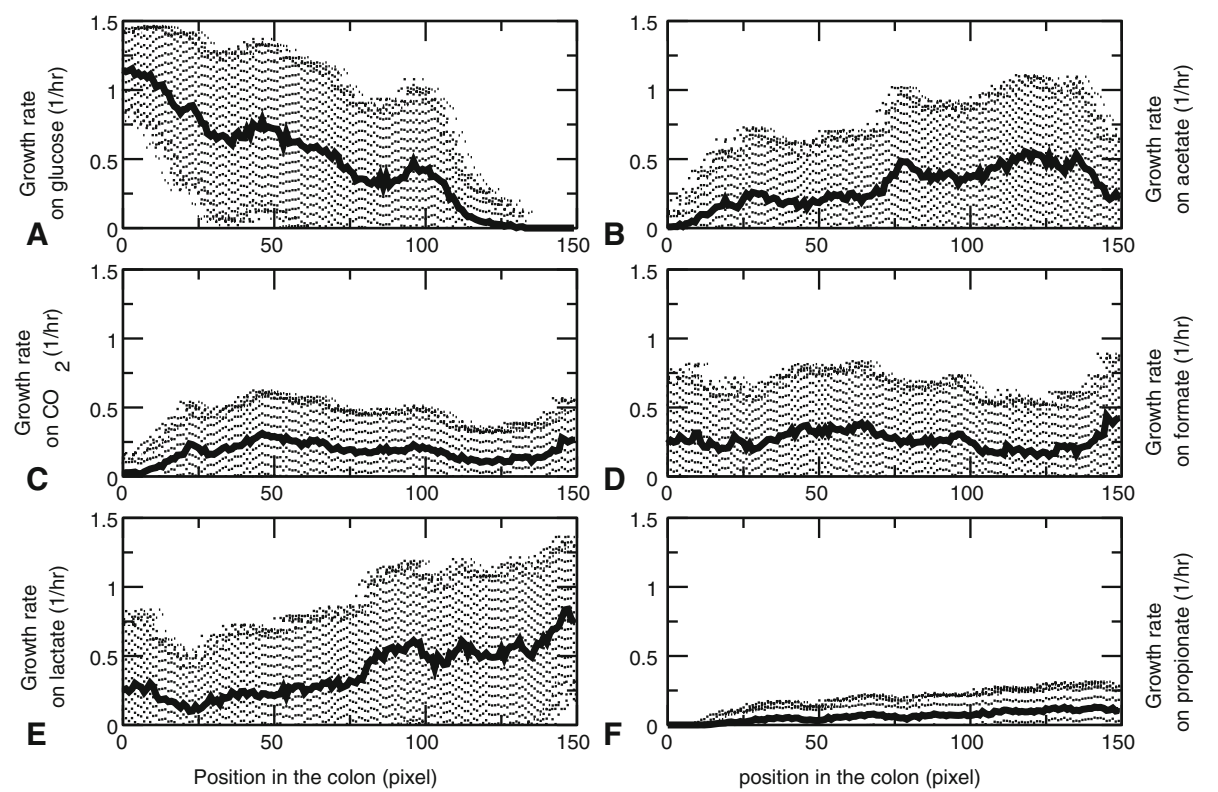

Fig. 7 Average growth rates along the colon. Average are taken over the final part of the simulations (3500-4000 hrs) All growth rates ar calculated in the presence of unlimited hydrogen gas, water, sodium, ammonium, phosphate, sulfate and protons. $\mathbf{a}$ Growth rate on glucose. $\mathbf{b}$ Growth rate on acetate. $\mathbf{c}$ Growth rate on $\mathrm{CO}_{2}$. $\mathbf{d}$ Growth rate on formate. e Growth rate on lactate. $\mathbf{f}$ Growth rate on propionate

stool samples from the Flemish and Dutch population [50] showed that, among a range of life-style related factors and medicine use, the diversity of the human gut microbiota correlates strongest with the Bristol stool scale (BSS), a self-assessed indicator of the "softness" of the stool. The analysis showed that for softer stools (higher stool index, indicative of faster transit times [51]), the diversity of the gut microbiota was reduced [52]. To investigate whether transit time could also be correlated with reduced diversity in our model, we studied the effect of increased fluxes through the tube ("diarrhea"), by assuming that the supra-bacteria flow through the tube at the same rate as the metabolites do. Strikingly, the maximal growth rate of the cells has become independent of the position (Fig. 9). Again, we clustered the population present at the end of the simulation with respect to their maximum growth rates in glucose, acetate, $\mathrm{H}_{2}$ and $\mathrm{CO}_{2}$, formate, lactate and propionate (Fig. 10). In contrast to the simulations without cell flow, the population does practically not diversify. All supra-bacteria can grow on glucose,

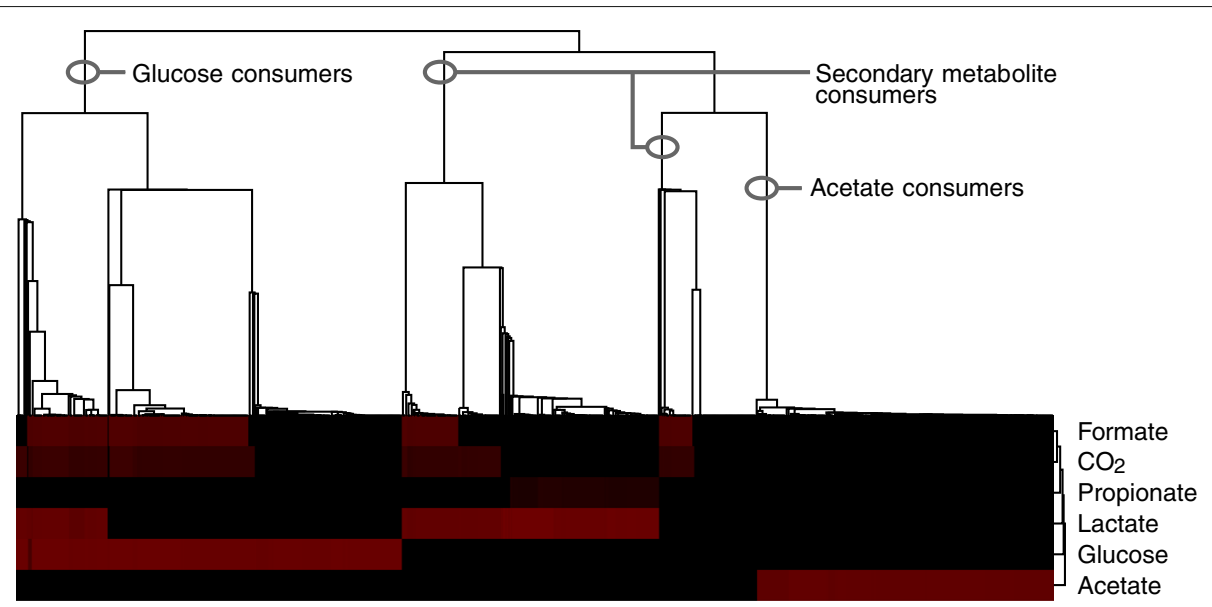

Fig. 8 Hierarchical clustering of all cells present at the end of the simulation, with respect to the growth rates on glucose, acetate, $\mathrm{CO}_{2}$, formate, lactate and propionate. Black indicates low growth rate, red high growth rate. We used [72] to perform the cluster analysis, with average linkage and a euclidian distance metric 

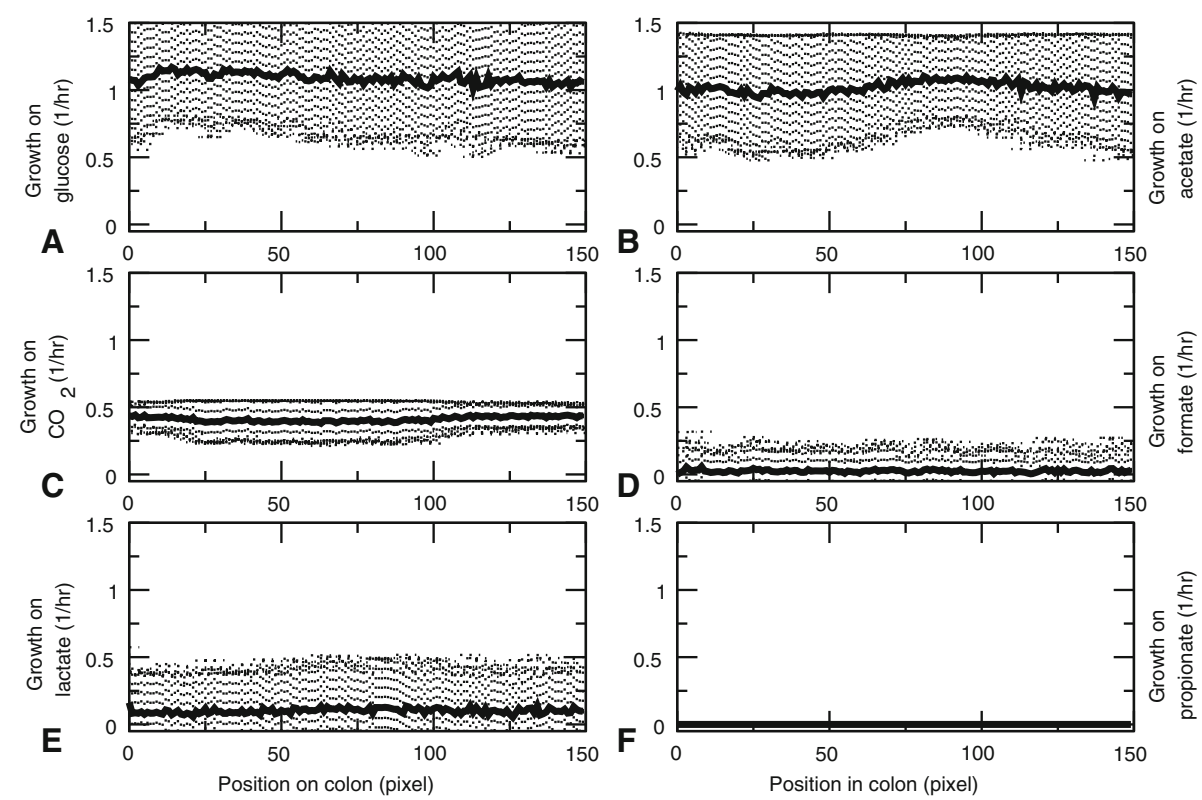

Fig. 9 Average growth rates along the colon, when cells flow through the colon as fast as metabolites. Average are taken over the final part of the simulations (3500-4000 hrs) All growth rates ar calculated in the presence of unlimited hydrogen gas, water, sodium, ammonium, phosphate, sulfate and protons. a Growth rate on glucose. b Growth rate on acetate. c Growth rate on $\mathrm{CO}_{2}$. $\mathbf{d}$ Growth rate on formate. e Growth rate on lactate.

f Growth rate on propionate

acetate and $\mathrm{H}_{2}$ and $\mathrm{CO}_{2}$. Thus, our simulations suggest that increased transit times may contribute to a reduction of microbial diversity, by reducing the spatial heterogeneity in the gut and, consequently, the construction of ecological niches and cross-feeding interactions.

\section{Discussion}

We have presented a coupled dynamic multi-species dynamic FBA and mass-transfer model of the gut microbiota. We first studied a non-spatial variant of the model, in order to determine to what extent cross-feeding can emerge in a non-evolving, diverse population of metabacteria. The individual metabacteria in this model contain the major carbohydrate fermentation pathways in the colon. Starting from glucose as a primary resource, the model produced acetate, butyrate, carbon dioxide, ethanol, formate, lactate and propionate. These fermentation products compared well with the short-chain fatty

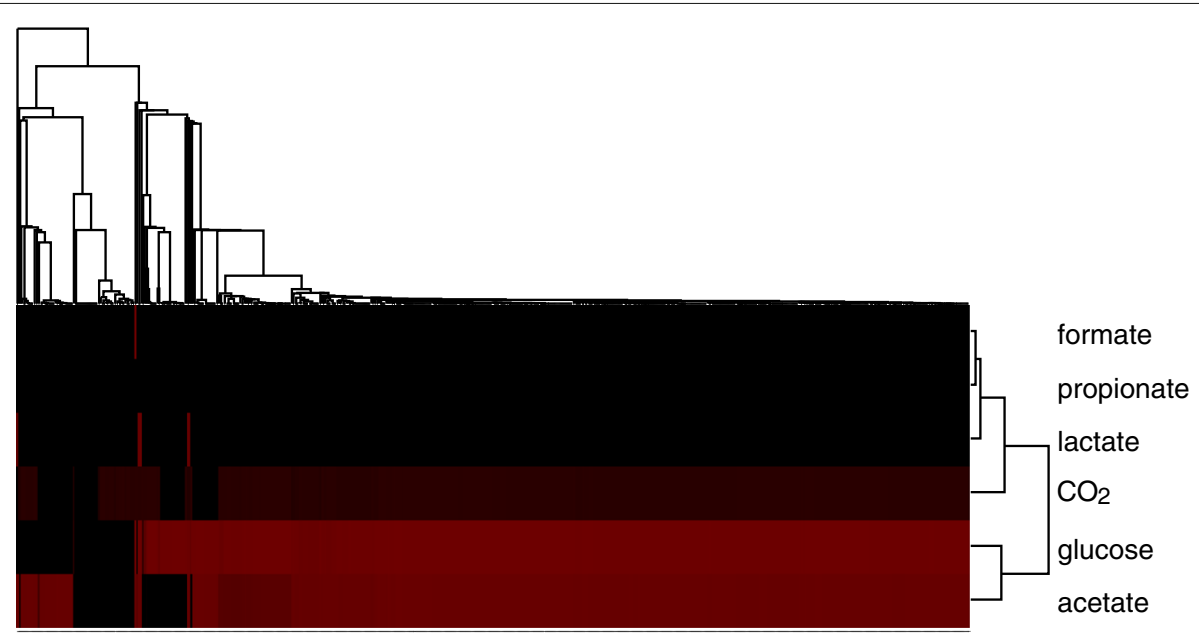

Fig. 10 Hierarchical clustering of all cells present at the end of the simulation with cell flow, with respect to the growth rates on formate, $\mathrm{CO}_{2}$, propionate, lactate, glucose and acetate. Black indicates low growth rate, red high growth rate. We used [72] to perform the cluster analysis, with average linkage and a euclidian distance metric 
acids found in the colon [37] or with those found in an in vitro model of the colon [38]. Our model generated these short-chain fatty acids only if it was run with FBAwMC and not with standard FBA, indicating that the individual metabacteria must be able to exhibit diauxic shifts. In FBAwMC these are due to rate-yield metabolic trade-offs [34, 36].

It has been argued that metabolic trade-offs in combination with mutational dynamics may already explain population diversity as it will select for suboptimal phenotypes with equally fit mutational neighbors - i.e., 'survival of the flattest' [53]. This mechanism may already sufficiently explain diversity in microbial ecosystems, suggesting that cross-feeding or spatial heterogeneity is not required for diversity. However, cross-feeding interactions exist in the gut $[54,55]$ and are likely to be an important factor in determining microbial diversity. Indeed, our spatially explicit, sdFBA model shows that already on a single food source a stratified structure of metabolic niches is formed, with glucose consumers in front, followed by strata inhabited by secondary and tertiary consumers.

Interestingly, these secondary and tertiary consumers specialized to their metabolic niche: Metabacteria sampled from the rear end of the tube could no longer grow on the primary resource glucose (Fig. 7a), and they grew better on the secondary metabolite lactate than bacteria from the front did (Fig. 7e). This specialization was mostly due to "gene loss", i.e., simplification of the metabolic networks. Interestingly, metabacteria with reduced genomes did not have a growth advantage in our model, yet they lost essential pathways required for metabolizing the primary resource. Such "trait loss without loss of function due to provision of resources by ecological interactions" [56] is indicative of an evolutionary mechanism known as compensated trait loss [56]. Note, however, that because smaller metabacteria did not have a growth advantage in our model, the gene loss in our model is due to drift. Hence it differs from the Black Queen Hypothesis [57], which proposes that the saving of resources associated with gene loss accelerate the evolution of compensated trait loss. An interesting future extension of the model would consider the metabolic costs associated with the maintenance of metabolic pathways.

The formation of metabolic niches and the observed compensated trait loss required that the metabacteria can maintain their approximate position in the gut-like tube, e.g., by adhering to the gut wall or by sufficiently fast reproduction [52]. The microbial diversification did not occur if the metabacteria moved along with the flow of the metabolites, a situation resembling diarrhea. Decreased microbial diversity is often seen causative for diarrhea, e.g., because it facilitates colonization by pathogenic species including Clostridium difficile [58]. Our model results suggest an additional, inverse causation, where accelerated transit reduces microbial diversity. Experimental studies are consistent with the idea that transit speed is causative for reduced diversity, but with a different mechanism: Microbiota sampled from softer stools (i.e., higher BSS and faster transit time) have higher growth potential, suggesting that faster transits favor fast growing species [52]. A second potential strategy to preventing wash-out from the gut at high transit times is adherence to the gut wall e.g., by the species of the $\mathrm{P}$ enterotype [52]. Thus these observations suggest that the reduction of microbial diversity at fast transits is due to selection for fast growing or adherent species. Our computational model suggests an alternative hypothesis, namely that increased transit times reduce the potential for bacterial cross-feeding, thus reducing the build-up of metabolic niches in the environment.

\section{Conclusion}

We have presented a coupled dynamic multi-species dynamic FBA and mass-transfer model of the gut microbiota. We first studied a non-spatial variant of the model, in order to determine to what extent cross-feeding can emerge in a non-evolving, diverse population of metabacteria. The individual metabacteria in this model contain the major carbohydrate fermentation pathways in the colon. Starting from glucose as a primary resource, the model produced acetate, butyrate, carbon dioxide, ethanol, formate, lactate and propionate. We next discussed a spatial variant of the model in a gut-like environment, a tube in which the metabolites diffuse and advect from input to output, and the bacteria attach to the gut wall. This spatially explicit, sdFBA model was extended with models of bacterial population dynamics, and 'mutation' of the metabacteria due to the gain and loss of pathways from the local population. In this model, a stratified structure of metabolic niches formed, with glucose consumers in front, followed by strata inhabited by secondary and tertiary consumers that lost the ability to grow on the primary resource. Interestingly, the stratification, and hence niche formation and specialization was lost if we increased transit speeds through the tube, to mimic diarrhea. Thus our model results suggest that enhanced enhanced transit speeds might contribute to the observation that softer stools (i.e., faster transit) have lower diversity [52].

Of course our model is a simplification as it lacks many key features of the gut microbiota and of the gut itself. The metabacterium only contain a minimal subset of the metabolic pathways that are found in the gut microbiota. Future versions of our model could extend the current metabacterium model with additional metabolic pathways, e.g., methanogenesis or sulfate reduction. Adding multiple pathways would increase the number of potential cross-feeding interactions and improve the biological 
realism of the model. An alternative route that we are currently taking is to include multiple, alternative metabacteria, each representing a functional group in the human gut microbiota [59]. This will allow us to compare the metabolic diversification observed in our computational model with metagenomics data, or use the model to compare alternative enterotypes [60].

A further simplification of this first study of our model, is that we have focused exclusively on glucose metabolism. Future versions of the model will also consider lipid and amino acid metabolism, allowing us to compare the effect of alternative "diets" and consider the break-down of complex polysaccharides present in plant-derived food fibers. Further extensions include more complex interactions with the gut wall, which is currently impenetrable as in some in vitro models of the gut microbiota $[61,62]$. Additional terms in Eq. 6 will allow us to study the effects of SCFA from the gut lumen, oxygen supply, and effects of the production of mucus by the gut wall [63].

\section{Methods}

\section{Metabolic model}

We converted the GEM of L. plantarum [28] to a stoichiometric matrix, $\mathbf{S}$. Reversible reactions were replaced by a forward and a backward irreversible reactions. Next, we added four metabolic pathways that are crucial in carbohydrate fermentation in the colon, but are not present in the network: propionate fermentation, butyrate fermentation, the acrylate pathway and the Wood-Ljungdahl pathway. We used the Kegg database (http://www.genome.jp/ kegg) [64] to add the necessary reactions. For the WoodLjungdahl pathway, we followed the review paper [49]. Additional file 6 lists all reactions and metabolites of the GEM, in particular those that we added to the GEM of L. plantarum.

To calculate the fluxes through the metabolic network as a function of the extracellular environment, we used fluxbalance analysis with molecular crowding (FBAwMC) $[34,35]$. FBAwMC assumes that all reactions through a are in steady state:

$$
\frac{d \vec{x}}{d t}=\mathbf{S} \cdot \vec{f}=0
$$

where $\vec{x}$ is a vector of all metabolites, $\vec{f}$ is a vector describing the metabolic flux through each reaction in the network, and $\mathbf{S}$ is the stoichiometric matrix. FBAwMC attempts to find a solution $\vec{f}$ of Eq. 8 that optimizes for an objective function under a set of constraints $\vec{f}_{\mathrm{b}} \leq \vec{f} \leq$ $\vec{f}_{\mathrm{ub}}$, with $\vec{f}_{\mathrm{lb}}$ and $\vec{f}_{\mathrm{ub}}$ the lower and upper bounds of the fluxes. Furthermore, FBAwMC constrains the amount of metabolic enzymes in the cell. This leads to the following constraint

$$
\sum a_{i} f_{i} \leq V_{\mathrm{prot}}
$$

where $a_{i} \equiv \frac{M v_{i}}{V b_{i}}$ is the "crowding coefficient", $M$ the cell mass, $V$ the cell volume, $v_{i}$ the molar volume of the enzyme catalysing reaction $i$ and $b_{i}$ is a parameter describing the proportionality between enzyme concentration and flux. For a derivation of Eq. 9 see Ref. [34]. $V_{\text {prot }}$ is a constant $\left(0 \leq V_{\text {prot }} \leq 1\right)$ representing the volume fraction of macromolecules devoted to metabolic enzymes. We use a value of $V_{\text {prot }}=0.2$, equal to the value used in [36] for other bacteria.

The crowding coefficients are not known for every reaction in the metabolic network. Therefore, following Vazquez and coworkers [35], crowding coefficients were chosen at random from a distribution of known crowding coefficients for $E$. coli based on published molar volumes (Metacyc [65]) and turnover numbers (Brenda [46]). Both in the well-mixed simulations as in the spatially explicit simulations, we allowed for unlimited influx of hydrogen gas, water, sodium, ammonium, phosphate, sulfate and protons. To calculate the growth rate, we find a solution of Eq. 8 that maximizes the rate of ATP production, given the crowding constraint (Eq. 9). ATP production has been shown to be a good proxy for biomass production [66] and it allows us to avoid the additional complexity of, e.g., amino acid metabolism and vitamin metabolism. The growth rate $\mu$ was then calculated by dividing the ATP production rate by a factor of 27.2, the factor that was used for ATP in the biomass equation of the original L. plantarum model [28].

\section{Well-mixed model}

Simulations of the well-mixed model are performed in Matlab, using the COBRA Toolbox [67]. We use an approach similar to Ref. [23] to model a population of cells in a well-mixed environment. We initiated 1000 cells with crowding coefficients for all their reactions set according to the experimental distribution of $E$. coli (see Section Metabolic model) We start with a total biomass concentration $(B)$ of 0.01 gram dry weight/liter (gDW/l), divided equally over all 1000 metabacteria (i.e., $\forall i \in$ $[1,1000]: B_{i}(0)=10^{-5}$ gr DW/l). At time $\mathrm{t}=0$ we initiate the environment with a glucose concentration of 1.0 $\mathrm{mM}$. At every time-step, the maximal uptake rate for each metabolite $j$ is a function of its concentration, $c_{j}(t)$, as,

$$
F_{\text {up, } \max }(j)=\frac{1}{\Delta t} \frac{c_{j}(t)}{\sum_{i=1}^{1000} B_{i}(t)} .
$$

We then perform FBAwMC for all 1000 supra-bacteria and update the concentrations of all metabolites that are excreted or taken up, as,

$$
c_{j}(t+\Delta t)=c_{j}(t)+\Delta t \sum_{i=1}^{1000} F_{i, j} B_{i}
$$


FBAwMC yields a growth rate $\mu_{i}$ for each suprabacterium $i$, which is used to update the biomass as,

$$
B_{i}(t+\Delta t)=B_{i}(t)+\mu_{i} B_{i}(t) \Delta t
$$

This procedure is continued until the supra-bacteria have stopped growing.

\section{Spatially explicit, evolutionary model}

For the spatially explicit simulations, we developed a $\mathrm{C}++$ package to perform constraint-based modeling using the GNU Linear Programming Kit (GLPK, http://www.gnu. org/software/glpk/) as linear programming tool. The multiscale, computational model of the gut microbiota was also developed in $\mathrm{C}++$. It describes individual metabacteria, or "cells" living on a grid, each with its own, unique GEM. Nutriets enter the grid at one end, flows through the grid, diffuses over the grid and is consumed by the cells. Uptake and excretion of metabolites is calculated using the GEM in each cell. The cells divide proportional to the calculated ATP production rate and mutate upon division. We simulate a total time of $4000 \mathrm{~h}$ (equivalent to 80000 time steps). A model description in pseudocode is given in Fig. 11. All parameters in the model are given in Table 1.

\section{Initialization}

We initialize the grid with cells that have the same metabolic network as in the well-mixed simulations. We choose the crowding coefficients for each reaction randomly. We allow maximally 2 cells to be present on each grid point. Thus, per grid point there are two "slots" that can be empty or filled by a cell. At time $t=0$, we initialize every slot of every grid point with a probability of $50 \%$ with a cell with random crowding coefficients. Because of the modeled population size (in the order of 1000 cells), each cell should be viewed as a metapopulation of bacteria that is representative for the local composition of the intestinal microbiota: i.e, a metabacterium.

\section{Nutrient dynamics}

We assumed that nutrients enter the colon every eight hours. In this study we consider glucose as the primary resource, because we want to focus on the bacterial diversity that can result from a single resource. Thus we assume that polysaccharides are already broken down to glucose. To allow for variability, we pick the amount of glucose from a normal distribution with mean of $42 \mathrm{mmol}$ and a relative standard deviation of $20 \%$. This mean value is chosen such that one the one hand all nutrients are consumed during passage through the gut and on the other hand it allows for a sufficiently large population size $(\approx 1000$ metabacteria).

The glucose is consumed by the metabacteria, according to the metabolic networks. These network take into

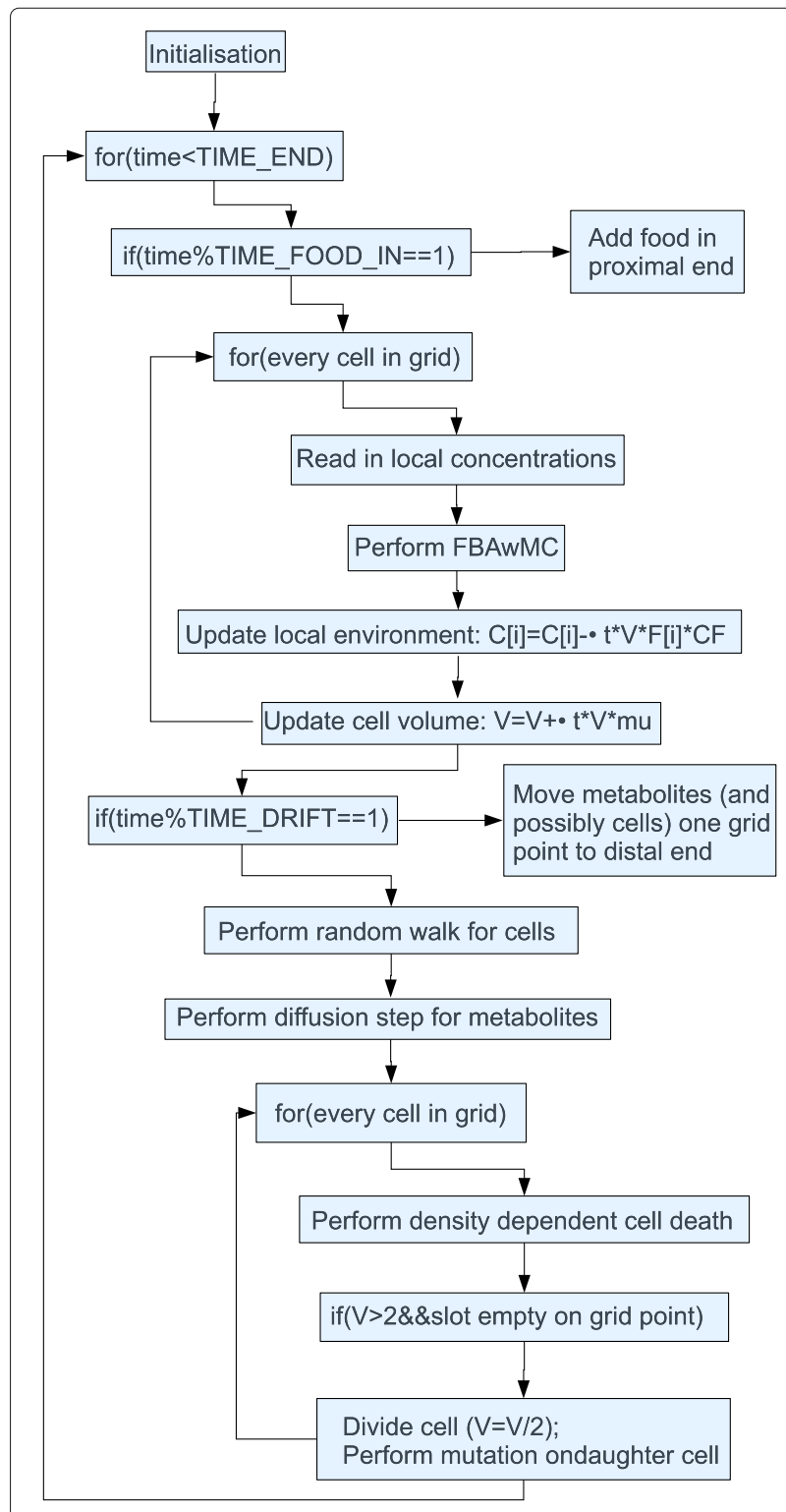

Fig. 11 Pseudocode of the spatially explicit computational model

account 115 extracellular metabolites, whose dynamics are all modeled explicitly in the model. The majority of these metabolites are never produced. Production and consumption for each metabolite is modeled using

$$
c_{i}(t+\Delta t)=c_{i}(t)+\Delta t \sum_{n=1}^{2}\left(F_{i, n} V_{n}\right. \text { DENS_MAX/4.0) }
$$

Thus, the concentration $c_{i}(t)$ of each metabolite $i$ is updated each timestep $\Delta t$ according to the calculated influx/efflux, $F_{i, n}$, and cell volume, $V_{n}$, of the cells on the grid point (maximally 2). Fluxes in the metabolic 
Table 1 Parameters of the spatially explicit model

\begin{tabular}{|c|c|c|c|}
\hline Parameter & Value & Units & Comments \\
\hline$\Delta \mathrm{t}$ & 3.0 & $\min$ & \\
\hline$\Delta x$ & 1.0 & $\mathrm{~cm}$ & \\
\hline Grid length & 150 & Grid sites & \\
\hline Grid height & 10 & Grid sites & \\
\hline TIME_END & 4000 & $\mathrm{hr}$ & \\
\hline \# slots per grid point & 2 & & \\
\hline DENS_MAX & 1.0 & $\left.\mathrm{~g} \mathrm{DW} \cdot\right|^{-1}$ & See main text \\
\hline Initial density & $50 \%$ & & Assumed \\
\hline TIME_FOOD & 8 & $\mathrm{hr}$ & Assumed \\
\hline FOOD_IN & 42 & $\mathrm{mmol}$ & Assumed \\
\hline Diffusion constant & 14000 & $\mu \mathrm{m}^{2} / \mathrm{s}$ & $\begin{array}{l}\text { Assumed (compare } \\
900 \mu \mathrm{m}^{2} / \mathrm{s} \text { glucose } \\
\text { in water) }\end{array}$ \\
\hline P_MOVECELL & 0.05 & & Assumed \\
\hline DEATH_BASAL & 0.025 & $h r^{-1}$ & Assumed \\
\hline DEATH_DENS & 2.0 & $h r^{-1}$ & Assumed \\
\hline TIME_DRIFT & 15 & $\min$ & $\begin{array}{l}\text { Passage time of } \\
\text { approximately } 40 \\
\text { hrs }\end{array}$ \\
\hline P_CELL_FLOW & Variable & & \\
\hline UPTAKE_HOST & Variable & & \\
\hline$\mu \_\mathrm{DEL}$ & 0.002 & & Assumed \\
\hline$\mu \_\mathrm{BIRTH}$ & 0.0002 & & Assumed \\
\hline$\mu \_\mathrm{POINT}$ & 0.002 & & Assumed \\
\hline$\mu \_P O I N T \_S T E P$ & 0.2 & & Assumed \\
\hline
\end{tabular}

network have unit $\mathrm{mmol} \cdot \mathrm{g} \mathrm{DW}^{-1} \cdot \mathrm{h}^{-1}$, where external metabolite concentrations are in $\mathrm{mmol} \cdot \mathrm{l}^{-1}$. To convert the fluxes to extracellular concentration changes, we therefore multiply with DENS_MAX; it is the maximum bacterial density in $\mathrm{g} \mathrm{DW} \cdot \mathrm{l}^{-1}$, which is estimated as explained in Table 1. The division by four is because there can be at maximum 2 cells of volume 2 at one grid point. DENS_MAX is the maximum local density of bacterial cells; it is used to calculate the change in metabolite concentration based on the metabolite influx and efflux. If a grid point is fully occupied with two meta-bacteria the cell density at that point equals DENS_MAX. A high DENS_MAX results in large changes in extracellular concentrations due to exchange fluxes. We estimated DENS_MAX using an estimated bacterial density of $10^{14}$ cells/l, an estimated bacterial cell size of $10^{-16} \mathrm{l} /$ cell and a cellular density of $100 \mathrm{~g} \mathrm{DW} / \mathrm{l}$, i.e., maxcelldensity $=10^{14} \frac{\text { cells }^{\prime}}{l} * 10^{-16} \frac{l}{\text { cells }} * 100 \frac{\mathrm{g} \mathrm{DW}}{\cdot}$ lcell $^{-1}$ $[68,69]$. To prevent negative concentrations, the uptake per time step $\Delta t$ is capped at

$$
\text { MAX_UPTAKE }_{i}=\frac{4.0 c_{i}}{\Delta t * \text { DENS_MAX } *\left(V_{1}+V_{2}\right)} .
$$

Each metabolite flows through the colon: Every $15 \mathrm{~min}$, all metabolites are shifted one grid point to the right. This results in a passage time of $37.5 \mathrm{~h}$, similar to observed colonic transit times (e.g., 39 hrs in [70]). Every metabolite is also dispersed uniformly due to turbulence and peristalsis. In absence of exact data for dispersion coefficients, we simplify these processes by a diffusion processes, with an effective diffusion constant of $14 \times 10^{3} \mu^{2} / \mathrm{s}$ for all metabolites. This dispersion coefficient is an order of magnitude higher than the diffusion constant of glucose in water, and provides a good balance between local mixing while maintaining sufficient differentiation in our simulations.

\section{Population dynamics}

FBAwMC yields growth rate, $\mu$, for each metabacterium $i$ using an empirical, auxiliary reaction [71]. The volume of the metabacterium is then updated, as

$$
V_{i}(t+\Delta t)=V_{i}(t)+V_{i}(t) * \mu_{i} * \Delta t .
$$

Cell death is taken into account in a density dependent way. This stabilizes the population, making sure that the population does not grow too fast if too much nutrients are given or dies out if too little nutrients are given. The death rate of a cell is calculated as follows

$$
\begin{aligned}
& \text { DEATH_RATE }=(\text { DEATH_BASAL }+ \text { DEATH_DENS } \\
& \left.\frac{\text { TOTAL_NEIGHBOURS }}{\text { MAX_NEIGHBOURS }}\right) \text {, }
\end{aligned}
$$

where TOTAL_NEIGHBOURS is the total amount of neighbours and MAX_NEIGHBOURS the maximum amount of neighbours (17 in the centre of the grid, because there are 2 slots per grid point).

Next the metabacteria expand into the empty patch on the same grid point when their volume exceeds a value of 2 . The volume of the parent metabacterium is then equally distributed over the two daughter metabacteria. During this expansion, three types of "mutations" can occur:

(a) the complete deletion of a reaction, i.e., extinction of the species responsible for this reaction, with probability $\mu \_D E L$;

(b) the reintroduction of metabolic pathways, corresponding to the invasion of the bacterium previously responsible for this pathway, with probability $\mu \_$BIRTH;

(c) the strengthening or weakening of one of the pathways, corresponding to the relative growth or suppression of a bacterial species in the metapopulation, with probability $\mu \_$POINT. 
To delete reaction (a) the maximal flux through that reaction is set to 0 . To reintroduce a reaction (b), we release the constraint by setting it to a practically infinite value $(999999 \mathrm{mmol} / \mathrm{gr} \mathrm{DW} \mathrm{hr}$ ). A point mutation (c) corresponds to a change of the crowding coefficient $\left(a_{i}\right.$ in Eq. 9) of that specific reaction, as

$$
a_{i, \text { new }}=a_{i, \text { old }} * 10^{\text {step }},
$$

In this way, the metabacteria specialize on certain reactions, i.e., by having only one or a few bacterial species in the patch. step is selected at random from a normal distribution with mean 0 and standard deviation $\mu \_P O I N T \_S T E P$. In this way, if the crowding coefficient is large, the mutation step will be large as well. This is necessary, because crowding coefficients are almost distributed log-normally $[35,36]$.

A possible non-physical side effect of this approach is that all crowding coefficients evolve to a value of $a_{i}=0$, in which case the growth rates would no longer be limited by enzymatic efficiency and volume of the patch. In reality, bacteria must trade off growth rate and growth yield (see Fig. 12 and Refs. [47, 48]). To take this tradeoff into account, we first calculate the total carbon uptake rate using FBAwMC as described above. We then calculate the maximal allowed growth rate, $\mu_{\max }$ belonging to that carbon uptake rate, using the empirical formula $\mu_{\max }=1 / 3.9 G_{\text {up }}$ (i.e., the black curve in Fig. 12). We cap the growth rate $\mu$ to the maximum growth rate, $\mu_{\max }$.

\section{Cell movement}

To model the cells' random movement over the grid, we loop over all grid points in random order. Every grid point has two "slots" that may or may not be occupied. Each

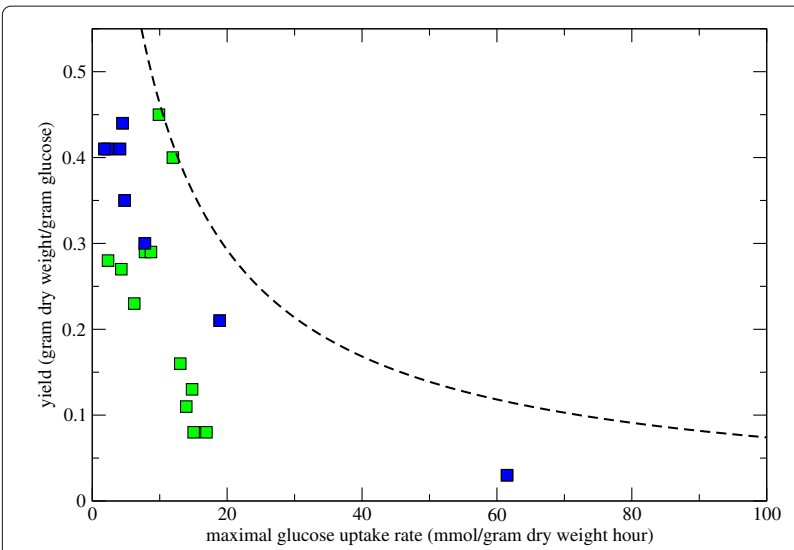

Fig. 12 Derivation of empirical formula for maximum growth rates as a function of the glucose uptake rate. Green squares are data from yeast species [48]; blue squares represent data from bacterial species [47]. The black, dashed curve is the maximum allowed growth yield given the glucose uptake rate, $G_{\text {up }}$. The empirical function is $\frac{1}{3.9 G_{u p}+2.8}$ and is designed such that all data points lie below it slot, whether it is occupied or not, has a probability of P_MOVECELL to exchange its position with a randomly chosen slot in a randomly chosen neighboring grid point, but this only succeeds if that slot has not already moved this turn.

An advection algorithm is introduced to model the flow of bacteria along the tube, with parameter P_CELL_ FLOW determining the advection velocity relative to the metabolite flux (see Section Nutrient dynamics). At each metabolite flow step (once every $15 \mathrm{~min}$ ), with probability P_CELL_FLOW all the cells shift one grid point to the right synchronously. I.e., for the default value P_CELL FLOW $=0$ the cells do not flow at all, whereas for P_CELL_ FLOW $=1$ the cells flow at the same rate as the metabolites. We performed simulations with P_CELL_FLOW $\in$ $\{0,0.5,1\}$. To mimic reentry of bacterial species from the environment, we assume periodic boundary conditions: All cells that leave the distal end of the gut, enter into the proximal end.

\section{Additional files}

Additional file 1: Figure S1. Simulation of the non-spatial, extended $L$. plantarum model using standard flux-balance analysis (FBA). Metabolite dynamics over time. The simulation is initialized with a pulse of glucose. Note that with standard FBA all 1000 cells behave identically, because the crowding coefficients are not used. (PDF $93 \mathrm{~kb}$ )

Additional file 2: Figure S2. Simulation of the non-spatial, standard $L$. plantarum model using flux-balance analysis with molecular crowding (FBAwMC). Metabolite dynamics over time. The simulation is initialized with a pulse of glucose. (PDF $105 \mathrm{~kb}$ )

Additional file 3: Figure S3. Population average and standard deviation of the cross-feeding factor $\mathrm{Ci}$ as a function of the position in the colon for all $n=10$ runs. The averages and standard deviation are over the vertical dimension and are calculated over the final part of the simulation, from 3500 h until 4000 h. (PDF 259 kb)

Additional file 4: Figure S4. Population averages of the metabolite concentrations over evolutionary time of the simulation in Fig. 5. (PDF $422 \mathrm{~kb}$ )

Additional file 5: Figure S5. Average metabolite concentraties along the tube for all $n=10$ simulations. The averages are taken over the second half of the simulations, from $2000 \mathrm{~h}$ to $4000 \mathrm{~h}$. (PDF $462 \mathrm{~kb}$ )

Additional file 6: Microsoft Excel File with all reactions and metabolites of the genome scale model of Lactobacillus plantarum [28], extended with proprionate fermentation, butyrate fermentation, the acrylate pathway, and the Wood-Ljungdahl pathway. (XLS 94 kb)

\section{Abbreviations}

BSS: Bristol stool scale; dFBA: Dynamic flux-balance analysis; DMMM: Dynamic multi-species metabolic model; FBA: Flux-balance analysis; FBAwMC: Flux-balance analysis with molecular crowding; GEM: GEnome-scale metabolic model; GLPK: GNU linear programming kit; ODE: Ordinary-differential equation; sdFBA: Spatial dynamic flux-balance analysis

\section{Acknowledgments}

The authors thank Daniël Muysken for his critical reading of the manuscript. We thank SURFsara (http://www.surfsara.nl) for the support in using the Lisa Compute Cluster.

\section{Funding}

This work was financed by the Netherlands Consortium for Systems Biology (NCSB), which is part of the Netherlands Genomics Initiative/Netherlands Organisation for Scientific Research. 


\section{Availability of data and material}

The dataset supporting the conclusions of this article is included within the article and its additional files.

\section{Authors' contributions}

$\mathrm{MvH}$ and RM designed the study and drafted the manuscript. MvH performed the simulations and analyzed the data. Both authors have read and approved the final version of the manuscript.

\section{Competing interests}

Milan J.A. van Hoek is Scientific Consultant for SysbioSim B.V. in Leiden, The Netherlands.

\section{Consent for publication}

Not applicable.

\section{Ethics approval and consent to participate}

Not applicable.

\section{Publisher's Note}

Springer Nature remains neutral with regard to jurisdictional claims in published maps and institutional affiliations.

\section{Received: 21 June 2016 Accepted: 26 April 2017}

Published online: 16 May 2017

\section{References}

1. Bäckhed F, Ley RE, Sonnenburg J, Peterson DA, Gordon Jl. Host-bacterial mutualism in the human intestine. Science. 2005;307(5717):1915-20. doi:10.1126/science.1104816.

2. Blaut M, Clavel T. Metabolic diversity of the intestinal microbiota: implications for health and disease. J Nutr. 2007;137(3 Suppl 2):751-5.

3. Greenblum S, Turnbaugh PJ, Borenstein E. Metagenomic systems biology of the human gut microbiome reveals topological shifts associated with obesity and inflammatory bowel disease. P Natl Acad Sci USA. 2012;109(2):594-9.

4. Durbán $A$, Abellán JJ, Jiménez-Hernández N, Artacho A, Garrigues $V$, Ortiz V, Ponce J, Latorre A, Moya A. Instability of the faecal microbiota in diarrhoea-predominant irritable bowel syndrome. FEMS Microbiol Ecol. 2013;86(3):581-9.

5. Rabiu BA, Gibson GR. Carbohydrates: a limit on bacterial diversity within the colon. Biol Rev Camb Philos Soc. 2002;77(3):443-53.

6. Cummings JH, Macfarlane GT. The control and consequences of bacterial fermentation in the human colon. J Appl Bacteriol. 1991;70(6):443-59.

7. De Filippo C, Cavalieri D, Di Paola M, Poullet JB, Massart S, Collini S, Pieraccini G, Lionetti P. Impact of diet in shaping gut microbiota revealed by a comparative study in children from Europe and rural Africa. P Natl Acad Sci USA. 2010;107(33):14691-6.

8. Helling RB, Vargas CN, Adams J. Evolution of Escherichia coli during growth in a constant environment. Genetics. 1987;116(3):349-58.

9. Ko EP, Yomo T, Urabe I. Dynamic clustering of bacteral population. Physica D. 1994;75:81-8.

10. Rosenzweig RF, Sharp RR, Treves DS, Adams J. Microbial evolution in a simple unstructured environment: genetic differentiation in Escherichia coli. Genetics. 1994;137(4):903-17.

11. Treves DS, Manning S, Adams J. Repeated evolution of an acetate-crossfeeding polymorphism in long-term populations of Escherichia coli. Mol Biol Evol. 1998;15(7):789-97.

12. Maharjan R, Seeto S, Notley-McRobb L, Ferenci T. Clonal adaptive radiation in a constant environment. Science. 2006;313(5786):514-7. doi:10.1126/science.1129865.

13. Kaneko K, Yomo T. Isologous diversification: a theory of cell differentiation. B Math Biol. 1997;59:139-96.

14. Kaneko K, Yomo T. Isologous Diversication for Robust Development of Cell Society. J Theor Biol. 1999;199:243-56.

15. Doebeli M. A model for the evolutionary dynamics of cross-feeding polymorphisms in microorganisms. Popul Ecol. 2002;44:59-70.

16. Pfeiffer T, Bonhoeffer S. Evolution of cross-feeding in microbial populations. Am Nat. 2004;163(6):126-35. doi:10.1086/383593.
17. Crombach A, Hogeweg P. Evolution of resource cycling in ecosystems and individuals. BMC Evol Biol. 2009;9:122. doi:10.1186/1471-2148-9-122.

18. Zomorrodi AR, Segrè D. Synthetic Ecology of Microbes: Mathematical Models and Applications. J Mol Biol. 2016;428(Part B):837-61.

19. Khandelwal RA, Olivier BG, Röling WFM, Teusink B, Bruggeman FJ. Community Flux Balance Analysis for Microbial Consortia at Balanced Growth. PLOS ONE. 2013;8(5):64567. doi:10.1371/journal.pone.0064567.s004.

20. Shoaie S, Ghaffari P, Kovatcheva-Datchary P, Mardinoglu A, Sen P, PujosGuillot E, de Wouters T, Juste C, Rizkalla S, Chilloux J, Hoyles L, Nicholson JK, Doré J, Dumas ME, Clement K, Bäckhed F, Nielsen J, Consortium MO. Quantifying Diet-Induced Metabolic Changes of the Human Gut Microbiome. Cell Metab. 2015;22(2):320-31. doi:10.1016/j.cmet. 2015.07.001.

21. Zomorrodi AR, Islam MM, Maranas CD. d-OptCom: Dynamic Multi-level and Multi-objective Metabolic Modeling of Microbial Communities. ACS Synth Biol. 2014;3(4):247-57. doi:10.1021/sb4001307.

22. Mahadevan R, Edwards JS, Doyle III FJ. Dynamic flux balance analysis of diauxic growth in Escherichia coli. Biophys J. 2002;83(3):1331-40.

23. Tzamali E, Poirazi P, Tollis IG, Reczko M. A computational exploration of bacterial metabolic diversity identifying metabolic interactions and growth-efficient strain communities. BMC Syst Biol. 2011;5:167. doi:10.1186/1752-0509-5-167.

24. Louca S, Doebeli M. Calibration and analysis of genome-based models for microbial ecology. eLife. 2015;4(e08208). doi:10.7554/eLife.08208.

25. Harcombe WR, Riehl WJ, Dukovski I, Granger BR, Betts A, Lang AH, Bonilla G, Kar A, Leiby N, Mehta P, Marx CJ, Segrè D. Metabolic resource allocation in individual microbes determines ecosystem interactions and spatial dynamics. Cell Rep. 2014;7(4):1104-15.

26. Cole JA, Kohler L, Hedhli J, Luthey-Schulten Z. Spatially-resolved metabolic cooperativity within dense bacterial colonies. BMC Syst Biol. 2015;9(1):395

27. Chen J, Gomez JA, Höffner K, Phalak P, Barton PI, Henson MA Spatiotemporal modeling of microbial metabolism. BMC Syst Biol. 2015;10(1):21-1.

28. Teusink B, Wiersma A, Molenaar D, Francke C, de Vos WM, Siezen RJ, Smid EJ. Analysis of growth of Lactobacillus plantarum wcfs1 on a complex medium using a genome-scale metabolic model. J Biol Chem. 2006;281(52):40041-8. doi:10.1074/jbc.M606263200.

29. Zhuang K, Izallalen M, Mouser P, Richter H, Risso C, Mahadevan R, Lovley DR. Genome-scale dynamic modeling of the competition between Rhodoferax and Geobacter in anoxic subsurface environments. ISME J. 2011;5(2):305-16.

30. Varma A, Palsson BO. Stoichiometric flux balance models quantitatively predict growth and metabolic by-product secretion in wild-type Escherichia coli W3110. Appl Environ Microb. 1994;60(10):3724-31.

31. Borenstein E. Computational systems biology and in silico modeling of the human microbiome. Brief Bioinform. 2012;13(6):769-80.

32. Pryde SE, Duncan SH, Hold GL, Stewart CS, Flint HJ. The microbiology of butyrate formation in the human colon. FEMS Microbiol Lett. 2002;217(2): 133-9.

33. Binsl TW, De Graaf AA, Venema K, Heringa J, Maathuis A, De Waard P, Van Beek JHGM. Measuring non-steady-state metabolic fluxes in starch-converting faecal microbiota in vitro. Benef Microb. 2010;1 (4): 391-405. doi:10.3920/BM2010.0038.

34. Beg QK, Vazquez A, Ernst J, de Menezes MA, Bar-Joseph Z, Barabasi AL, Oltvai ZN. Intracellular crowding defines the mode and sequence of substrate uptake by Escherichia coli and constrains its metabolic activity. P Natl Acad Sci USA. 2007;104(31):12663-8. doi:10.1073/pnas.0609845104.

35. Vazquez A, Beg QK, Demenezes MA, Ernst J, Bar-Joseph Z, Barabasi AL, Boros LG, Oltvai ZN. Impact of the solvent capacity constraint on e. coli metabolism. BMC Syst Biol. 2008;2:7. doi:10.1186/1752-0509-2-7.

36. van Hoek MJA, Merks RMH. Redox balance is key to explaining full vs. partial switching to low-yield metabolism. BMC Syst Biol. 2012;6(1):22. doi:10.1186/1752-0509-6-22.

37. Cummings JH, Pomare EW, Branch WJ, Naylor CP, Macfarlane GT. Short chain fatty acids in human large intestine, portal, hepatic and venous blood. Gut. 1987;28(10):1221-7.

38. de Graaf AA, Maathuis A, de Waard P, Deutz NEP, Dijkema C, de Vos WM, Venema K. Profiling human gut bacterial metabolism and its kinetics using [u-13c]glucose and nmr. NMR Biomed. 2010;23(1):2-12. doi:10.1002/nbm.1418. 
39. Price ND, Reed JL, Palsson BO. Genome-scale models of microbial cells: evaluating the consequences of constraints. Nat Rev Microbiol. 2004;2(11):886-97.

40. Moens F, Verce M, De Vuyst L. Lactate- and acetate-based cross-feeding interactions between selected strains of lactobacilli, bifidobacteria and colon bacteria in the presence of inulin-type fructans. Int J Food Microbiol. 2017;241:225-36.

41. den Besten G, Lange K, Havinga R, van Dijk TH, Gerding A, van Eunen K, Muller M, Groen AK, Hooiveld GJ, Bakker BM, Reijngoud DJ. Gut-derived short-chain fatty acids are vividly assimilated into host carbohydrates and lipids. AJP Gastrointest Liver Physiol. 2013;305(12):900-10.

42. Pfeiffer T, Schuster S, Bonhoeffer S. Cooperation and competition in the evolution of ATP-producing pathways. Science. 2001;292(5516):504-7.

43. Venema K, van den Abbeele P. Experimental models of the gut microbiome. Best Pract Res Cl Ga. 2013;27(1):115-26.

44. Minekus M, Smeets-Peeters M, Bernalier A, Marol-Bonnin S, Havenaar R, Marteau P, Alric M, Fonty G, Huis in't Veld JH. A computer-controlled system to simulate conditions of the large intestine with peristaltic mixing, water absorption and absorption of fermentation products. Appl Microbiol Biot. 1999;53(1):108-14.

45. Macfarlane S, Woodmansey EJ, Macfarlane GT. Colonization of Mucin by Human Intestinal Bacteria and Establishment of Biofilm Communities in a Two-Stage Continuous Culture System. Appl Environ Microb. 2005;71(11): 7483-92.

46. Chang A, Scheer M, Grote A, Schomburg I, Schomburg D. Brenda, amenda and frenda the enzyme information system: new content and tools in 2009. Nucleic Acids Res. 2009;37(Database issue):588-92. doi:10.1093/nar/gkn820.

47. Fuhrer T, Fischer E, Sauer U. Experimental identification and quantification of glucose metabolism in seven bacterial species. J Bacteriol. 2005;187(5):1581-90. doi:10.1128/JB.187.5.1581-1590.2005.

48. Merico A, Sulo P, Piskur J, Compagno C. Fermentative lifestyle in yeasts belonging to the Saccharomyces complex. FEBS J. 2007;274(4):976-89.

49. Ragsdale SW, Pierce E. Acetogenesis and the wood-ljungdahl pathway of co(2) fixation. Biochim Biophys Acta. 2008;1784(12):1873-98. doi:10.1016/j.bbapap.2008.08.012.

50. Falony G, Joossens M, Vieira-Silva S, Wang J, Darzi Y, Faust K, Kurilshikov A, Bonder MJ, Valles-Volomer M, Vandeputte D, Tito RY, Chaffron S, Rymenans L, Verspecht C, De Sutter L, Lima-Mendez G, D’hoe K, Jonckheere K, Homola D, Garcia R, Tigchelaar EF, Eeckhaudt L, Fu J, Henckaerts L, Zhernakova A, Wijmenga C, Raes J. Population-level analysis of gut microbiome variation. Science. 2016;352(6285):560-4.

51. Lewis SJ, Heaton KW. Stool Form Scale as a Useful Guide to Intestinal Transit Time. Scand J Gastroentero. 1997;32(9):920-4.

52. Vandeputte D, Falony G, Vieira-Silva S, Tito RY, Joossens M, Raes J. Stool consistency is strongly associated with gut microbiota richness and composition, enterotypes and bacterial growth rates. Gut. 2015;65(1): 57-62.

53. Beardmore RE, Gudelj I, Lipson DA, Hurst LD. Metabolic trade-offs and the maintenance of the fittest and the flattest. Nature. 2011;472(7343): 342-6. doi:10.1038/nature09905.

54. Falony G, Vlachou A, Verbrugghe K, De Vuyst L. Cross-feeding between bifidobacterium longum bb536 and acetate-converting, butyrate-producing colon bacteria during growth on oligofructose. Appl Environ Microb. 2006;72(12):7835-41. doi:10.1128/AEM.01296-06.

55. Flint HJ, Duncan SH, Scott KP, Louis P. Interactions and competition within the microbial community of the human colon: links between diet and health. Environ Microbiol. 2007;9(5):1101-11. doi:10.1111/j. 1462-2920.2007.01281.x.

56. Ellers J, Toby Kiers E, Currie CR, McDonald BR, Visser B. Ecological interactions drive evolutionary loss of traits. Ecol Lett. 2012;15(10): 1071-82.

57. Morris JJ, Lenski RE, Zinser ER. The Black Queen Hypothesis: evolution of dependencies through adaptive gene loss. MBio. 2012;3(2):e00036-12.

58. Chang JY, Antonopoulos DA, Kalra A, Tonelli A, Khalife WT, Schmidt TM, Young VB. Decreased Diversity of the Fecal Microbiome in Recurrent Clostridium difficile-Associated Diarrhea. J Infect Dis. 2008;197(3):435-8.

59. Gill SR, Pop M, Deboy RT, Eckburg PB, Turnbaugh PJ, Samuel BS, Gordon JI, Relman DA, Fraser-Liggett CM, Nelson KE. Metagenomic analysis of the human distal gut microbiome. Science. 2006;312(5778): 1355-9. doi:10.1126/science.1124234.
60. Arumugam M, Raes J, Pelletier E, Le Paslier D, Yamada T, Mende DR, Fernandes GR, Tap J, Bruls T, Batto JM, Bertalan M, Borruel N, Casellas F, Fernandez L, Gautier L, Hansen T, Hattori M, Hayashi T, Kleerebezem M, Kurokawa K, Leclerc M, Levenez F, Manichanh C, Nielsen HB, Nielsen T, Pons N, Poulain J, Qin J, Sicheritz-Ponten T, Tims S, Torrents D, Ugarte E, Zoetendal EG, Wang J, Guarner F, Pedersen O, de Vos WM, Brunak S, Doré J, Antolín M, Artiguenave F, Blottiere HM, Almeida M, Brechot C, Cara C, Chervaux C, Cultrone A, Delorme C, Denariaz G, Dervyn R, Foerstner KU, Friss C, van de Guchte M, Guedon E, Haimet F, Huber W, van Hylckama-Vlieg J, Jamet A, Juste C, Kaci G, Knol J, Lakhdari O, Layec S, Le Roux K, Maguin E, Mérieux A, Melo Minardi R, M'rini C, Muller J, Oozeer R, Parkhill J, Renault P, Rescigno M, Sanchez N, Sunagawa S, Torrejon A, Turner K, Vandemeulebrouck G, Varela E, Winogradsky Y, Zeller G, Weissenbach J, Ehrlich SD, Bork P. Enterotypes of the human gut microbiome. Nature. 2011;473(7346):174-80.

61. Molly K, Vande Woestyne M, Verstraete W. Development of a 5-step multi-chamber reactor as a simulation of the human intestinal microbia ecosystem. Appl Microbiol Biot. 1993;39(2):254-8.

62. Molly K, Woestyne MV, Smet ID. Validation of the simulator of the human intestinal microbial ecosystem (SHIME) reactor using microorganism-associated activities. Microb Ecol Health D. 1994;7(4): $191-200$.

63. Kashyap PC, Marcobal A, Ursell LK. Genetically dictated change in host mucus carbohydrate landscape exerts a diet-dependent effect on the gut microbiota. P Natl Acad Sci USA. 2013;110(42):17059-64.

64. Kanehisa M, Goto S, Sato Y, Furumichi M, Tanabe M. Kegg for integration and interpretation of large-scale molecular data sets. Nucleic Acids Res. 2011;40(Database issue):D109-14.

65. Caspi R, Foerster H, Fulcher CA, Kaipa P, Krummenacker M, Latendresse M, Paley S, Rhee SY, Shearer AG, Tissier C, Walk TC, Zhang P, Karp PD. The metacyc database of metabolic pathways and enzymes and the biocyc collection of pathway/genome databases. Nucleic Acids Res. 2008;36(Database issue):623-31. doi:10.1093/nar/gkm900.

66. Schuetz R, Kuepfer L, Sauer U. Systematic evaluation of objective functions for predicting intracellular fluxes in Escherichia coli. Mol Syst Biol. 2007;3:119. doi:10.1038/msb4100162.

67. Schellenberger J, Que R, Fleming RMT, Thiele I, Orth JD, Feist AM, Zielinski DC, Bordbar A, Lewis NE, Rahmanian S, Kang J, Hyduke DR, Palsson BO. Quantitative prediction of cellular metabolism with constraint-based models: the cobra toolbox v2.0. Nat Protoc. 2011;6(9): 1290-307. doi:10.1038/nprot.2011.308.

68. Norland S, Heldal OM, AND and Tumyr. On the relation between dry matter and volume of bacteria. Microbial Ecol. 1987;13:95-101.

69. Ley RE, Peterson DA, Gordon J. Ecological and evolutionary forces shaping microbial diversity in the human intestine. Cell. 2006;124(4): 837-48. doi:10.1016/j.cell.2006.02.017.

70. Arhan P, Devroede G, Jehannin B, Lanza M, Faverdin C, Dornic C, Persoz B, Tétreault L, Perey B, Pellerin D. Segmental colonic transit time. Dis Colon Rectum. 1981;24(8):625-9.

71. Orth JD, Thiele I, Palsson B $\varnothing$. What is flux balance analysis? Nat Biotechnol. 2010;28(3):245-8.

72. Guffanti A. Finding the needle in the haystack. Genome Biol. 2002;3: reports2008. doi:10.1186/gb-2002-3-2-reports2008. 